\title{
Digital outline of elementary particles via a root space diagram approach
}

\author{
Erik Trell \\ Faculty of Health Sciences, University of Linköping, Se-581 83 Linköping, Sweden \\ E-mail: erik.trell@gmail.co
}

\begin{abstract}
In the immediate intuitive as well as penetrating logical contemplation, the universe stands forth as an ever-present entity; consisting solely of itself, and fulfilling solely itself. A truism and a truth, and also a conclusion by proxy because its shape expressions are covered by curvilinear mathematical functions which completely exhaust their solution spaces, displacing any 'parallel' universes to isolates of less perfection since the fundamental formative categories of straight and round are thereby already consummated by our version. My first impression, under the northern light upon the velvet screen in starry polar nights of childhood Lapland, still holds, then, of the shining moon continuously swirling along with me and everything else from our preceding moment into our next, with the advancing now-slice flanked by the blackened archive of used and pending slices always restoring precisely enough room for the whole past and future at the one and same time. This vision and its lingering question may here be resolved by a facts and findings-based scientific testing which I feel is facilitated by my meantime experience as a rational researcher in a related field of descriptive natural history as well as by another archetypal arctic apprehension hard to miss when witnessing the instant change of water to ice from hence isotropically dispersed origins at once inflated over the entire surface of a freezing lake: it is a phase transition, where one uniform element alone is simultaneously spanned into an alternative principal form of space-filling alignment and where dynamics flares up in that timeless quantum fluctuation. Like the partial derivatives of differential equations the irreducible unit in question is the infinitesimal straight line undergoing the classical three-dimensional Euclidean space Lie algebra transformation of its orientation "between the Plücker line geometry and a geometry whose elements are the space's spheres." Coming back later to the ontology and prescribed machinery of this, the fully and easily reproducible outcome comprises the complete observed elementary particle spectroscopy with detailed mass numbers, charge levels and angular momentums organized in a continuously space-filling polyhedral space frame lattice architecture that over sequential levels of self-expansion meets up with presently disclosed nanotechnological structures so as to directly making up matter in a binary digital mode which is essentially that of dialectics and logic, too, consorting the Cartesian cut between concrete and abstract; matter and mind; structure and language from the very start. As actual realization as well as computational method, the only, and prior, and equally definite and uncircumventable parallel is professor Santilli's revolutionary iso-. geno- and hypermathematics, whereas for structure the only parallel to straight is-straight, leaving scant latitude at least for a simpler workable universe.
\end{abstract}

Keywords: Antiparticles, atom honeycombs, computational method, digital universe, elementary particle spectroscopy, Euclidean space, hadronic mechanics, information systems, Lie algebra realization, mass numbers, nanotechnology, periodic system, phase transition, quarks, regular solids, truncated octahedron

PACS: 02.20.Qs, 02.40.Dr, 07.05.Bx, 07.05.Tp, 11.15.Ha, 12.10.Kt, 12.39.Ba, 12.40.Yx, 14.20.-c, 14.40.-n, 14.60.-z

\section{Introduction}

Standing here before so many learned colleagues at this avantgarde summit and bestowed with the great honour of delivering its inauguration speech, I am truly filled with gratitude and joy, but also with the slightly embarrassing awareness of the risk of tiring you with the same lecture again and again over the years. I therefore happily grasp the kind suggestion of my great friend and mentor, professor

1472-7978/13/\$27.50 (C) 2013 - IOS Press and the authors. All rights reserved

This article is published online with Open Access and distributed under the terms of the Creative Commons Attribution NonCommercial License. 


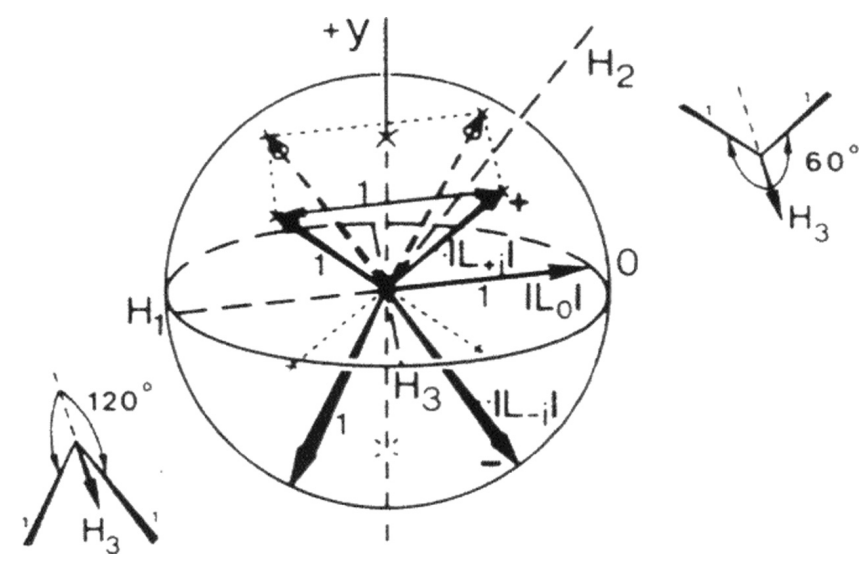

Fig. 1. Real form three-dimensional spherical Lie algebra neighbourhood with duplicated $\mathrm{A}_{2}$ root space diagrams.

Ruggero Maria Santilli, to make a kind of phase transition myself, that is, to reverse the synopsis in tune with its so to say personal history, that is, my stumbling way to at least some kind of own understanding. Because it started out in a feeling long ago, a sense of coherence as it were, with the moon and the stars in frozen moments amplified by snowy reflection in the polar winter-nights of childhood Lapland. I felt the celestial timelessness of their glazed illumination, where the hollow space was not empty as it appeared but filled by past and future continuously exchanged in the perpetual progress of a single flaring slice of Now, constantly rounded off in three sufficient dimensions both in and of that endless cosmic ice-block. And this has remained the lay-out and plan of my pursuit of the actual constitution of real matter "from the smallest to the largest scale" [1] in a communion and interplay of complementary rectangular and spherical symmetry, each quiescent and inert without the other but between them spanning all the forms and shapes that we encounter and are part of in our equally abstract as concrete world. The report will be descriptive with emphasis upon the reproducible objective results, which remain as the easily testable authentic facts and findings they are in the lasting scientific records. They combine the foremost classical methods and models with recent developments like inflation [Ib.] and loop quantum gravity [2], and their extra strength is the full compatibility and lining up with modern Nanotechnology, fitting it with a selfsimilar building kit and digital computational and exposition method of real structures at the infinitesimal level.

\section{Methods and results}

\subsection{The baryons}

There were other reasons, too, to go for round, but I may come back to that later. In any case, the first

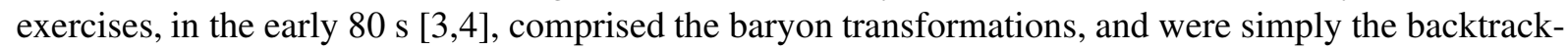
ing of their scattering experiments mapped in the canonical $\mathrm{SO}(3) \times \mathrm{O}(5)$ geometrical automorphism of Lie algebra SU(3). (Fig. 1)

The figure is not ad hoc as it might seem, but based on a mathematical demonstration [5] that since the flat $\mathrm{A}_{2}$ diagram is over the double-valued complex plane, its representation over the actual parent real space imposes this duplication into and continuing outside (Fig. 2) of the spherical envelope. Furthermore it is how Lie himself in his original thesis projected his differential transformations "between 


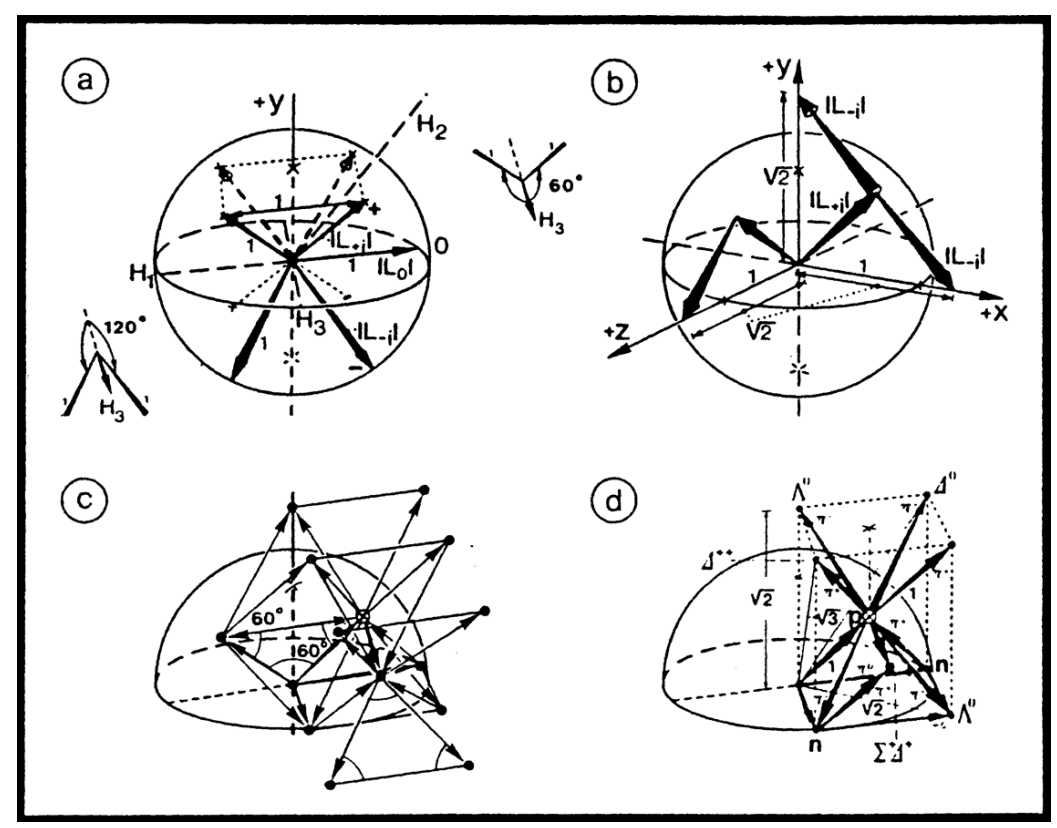

Fig. 2. Spherical root vector space whose neutral $t$ isospin root vectors coincide with Cartesian $\mathrm{X}$ and $\mathrm{Z}$ axes (a) but whose charged $t$ isospin axes (b) set up a non-commutative quark matrix with unit side (c), continuing in the global interstice as space-filling regular tetra- and octahedrons, (d) none of which can fill the space separately due to different side length in lateral and bottom planes. It is seen that it is the vertical side that is non-commutating with the cubical framework, so that it is this virtual fall of the phase transition which from its first extra-nucleon evolution cycle becomes more and more irreversible.

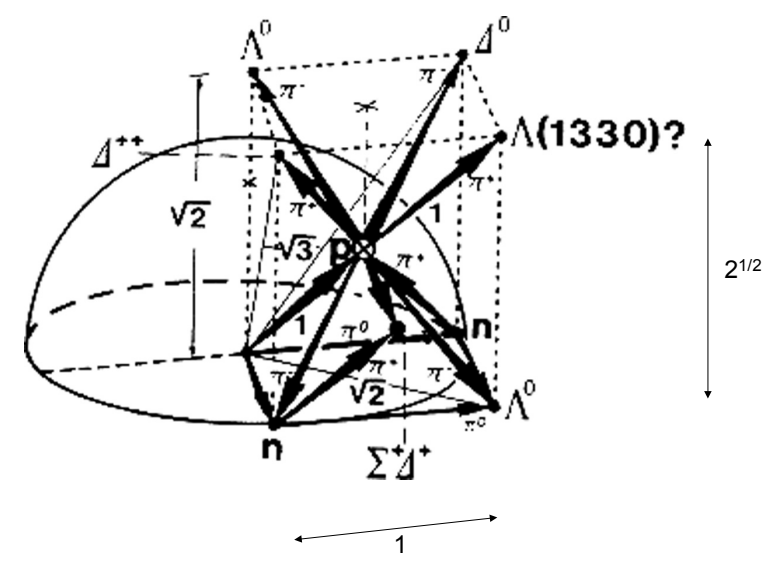

Fig. 3. Diagram of first and second layer of lattice formed by the unit length $A_{2}$ root vectors in one of the eight over diagonals mirrored Cartesian space segments with major semiaxis of ellipsoidal transformation from outer end-points delineated in $\Lambda^{0}$ (length $=\left[1^{2}+1^{2}\right]^{1 / 2}$ and $\Delta^{0}$ and $\Delta^{++}\left(\left[1^{2}+\left(2^{1 / 2}\right)^{2}\right]^{1 / 2}\right)$ in both) while inferable in the others $\left(\Sigma^{+}, \Delta^{+}\right.$and $\left.\Delta^{-}\right)$.

the Plücker line geometry and a geometry whose elements are the space's spheres" [6,7]. And finally it is a faithful three-dimensional execution of the "eightfold way" of Gell-Mann and Neeman, sharing its identification of the unit $t$ isospin root vectors with the pion channels, and hence realising an "eightfold eightfold way" $[8,9]$ with extensive theoretical and methodological authenticity and underpinning. Figure 3 exemplifies the procedure in closer detail. The neutral and charged $t$ isospin unit pion steps 
are seen to fan out to the respective positions in the next lattice level and thus, just as the hypercharge vectors in the plane eightfold way diagram pinpoint the preferred major semiaxis locations of resulting transformations of the state they arise from. Like in the actual scattering experiments other orientations may occur, too, giving an equal background horizon of random events as in reality, but the former would be the sharper peaks. Again this is a striking correspondence with observations, as is the case also with all other recorded properties including faithful geometrical representation of the gauge and color invariance of quantum chromodynamics by the volume and spheroidal symmetry preservation of the series of ellipsoidal transformations from the ground nucleon sphere and onwards that naturally come about in this way [10, pp. 404-405].

One sees that the shortest major semiaxis change results from a $\pi^{0}$ isospin step from the $\mathrm{n}$ (neutron) and $\pi^{-}$from the $\mathrm{p}$ (proton) surface points of the sphere, converging at the (until then anticipated) $\Lambda^{0}$ endpoint in the neutral equatorial plane. Clearly, a line from there to the nucleon centre, projecting the major semiaxis of the respective ellipsoid transformation will have length $2^{1 / 2}$ in the unit scale, and when the volume of the sphere is preserved, the minor semiaxis in the most likely symmetrical execution will have length $1 / 2^{1 / 4}$, from which the mass relative to the spherical ground-state can be accurately calculated as described below. Also the end-point of $\Sigma^{+}$by a $\pi^{0}$ from $\mathrm{p}$ and a $\pi^{+}$from $\mathrm{n}$ can be traced but its major semiaxis projection to the nucleon north pole is not outlined here. Likewise not shown, the major semiaxis projection from this point might alternatively be to the nucleon centre with resulting length $3^{1 / 2}$ and consequential minor semiaxis in a symmetrical transformation $1 / 3^{1 / 4}$, and this variety is the $\Delta^{+} . \Delta^{++}$by a $\pi^{+}$from $\mathrm{p}$ and major semiaxis $3^{1 / 2}$ and $\Delta^{0}$ by a $\pi^{-}$from $\mathrm{p}$ also with major semiaxis $3^{1 / 2}$ are easily calculable as drawn, and a $\Delta^{-}$by a $\pi^{-}$from $\mathrm{n}$ can be inferred. With identical channels and charge levels, the $\Lambda, \Sigma$ and $\Delta$ hyperons are the first ones seen in the real spectroscopy, too, so that the extensive correspondence with reality is established right from the initial steps.

It is interesting that the duplicated $\mathrm{A}_{2}$ root space vectors form a three-dimensional coordinate system connecting to an endless hexagonal lattice which is diagonal to the Euclidean space and linearly independent from this and so realising the $u, d, s$ quark dimensionalities in ordinary geometry. Only the mass, or energy levels are lacking now, but they are also intrinsic and immediately retrievable and possible to compute in the transformations by considering their identification with the minor semiaxis, i.e. compression of these according to the quark pressure formula, $\Delta p=\hbar / \Delta x$ [11], making them inversely proportional by that length to the mass of the perfectly spherical proton with all semiaxes $=1[3-5,8,9$, $12,13]$.

In Fig. 4, the plane graphs summarize the channels and the major semiaxis endpoints arrived at in the $\Lambda^{0}, \Sigma^{+, 0,-}$ and $\Delta^{++,+, 0,-}$ hyperons with lengths to the origin given by the root expression, and further that also the charge levels are retrieved exactly and exhaustively as in reality. The global, quark-skewed hexagonal spherical root space lattice is shown in the p-n transposition and (the equatorial plane of) the volume-preserving ellipsoidal reconfiguration is shown in the $\Lambda^{0}$ state.

In Fig. 5, the plane graphs summarize the channels and the major semiaxis endpoints arrived at in the ensuing $\Xi^{-, 0}, \Sigma(1385)^{+,-}$and $\Lambda(1405)^{0}$ hyperons, likewise with lengths to the origin given by the root expressions. It is seen that also these states come out exactly and exhaustively in all their channel, charge, calculable mass and also $\mathrm{J}^{\mathrm{p}}$ (by levels out from the ground state) numbers as in reality.

Figure 6 summarizes the shape of all the states in the original eightfold way supermultiplets as obtained from first possible consecutive steps in the root vector lattice with major semiaxis between their endpoints and underlying states, and minor semiaxis derived from the volume and symmetry preservation condition.

And Table 1 shows the results of the direct computation method of the masses in the same states. 


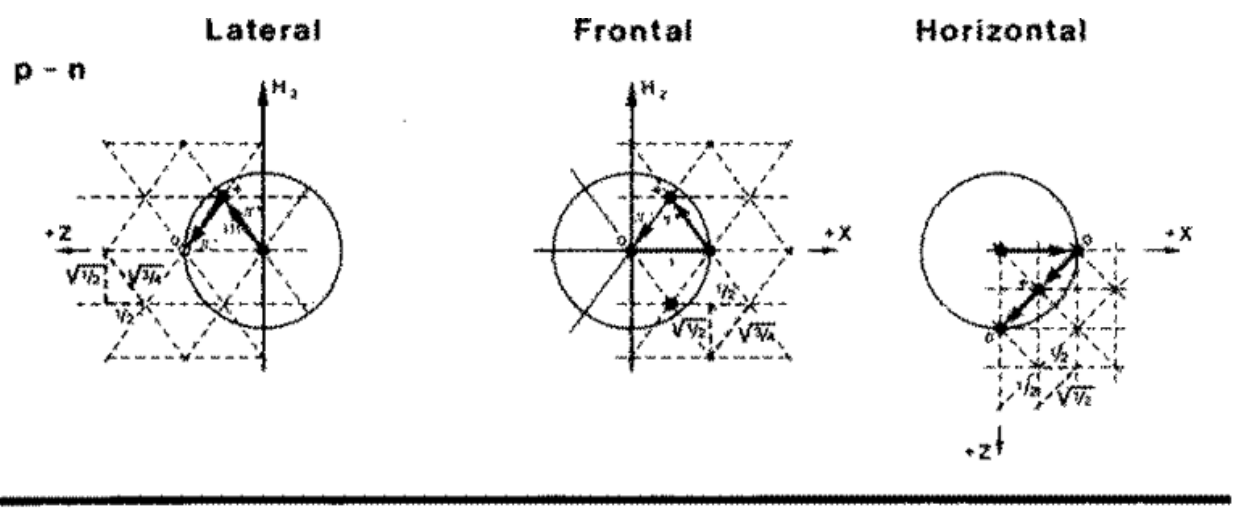

( $A^{3}$
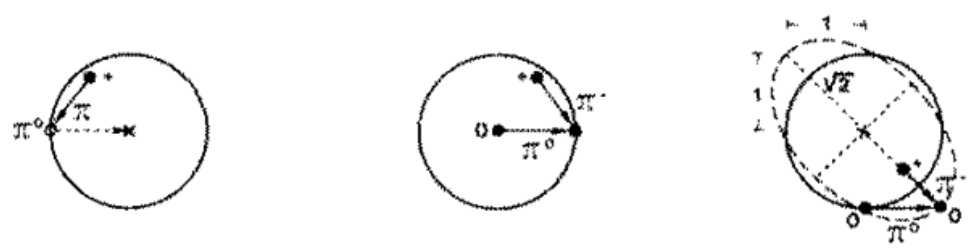

(2)
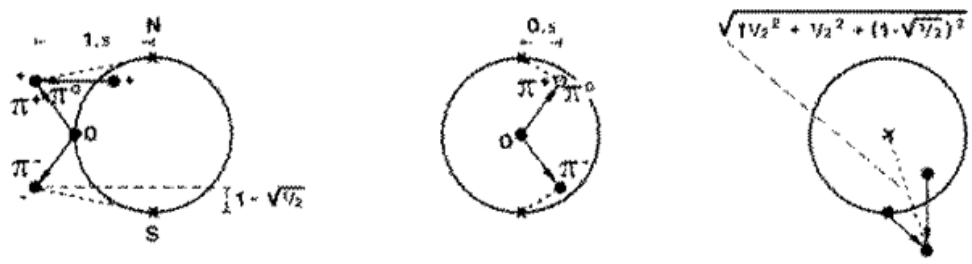

(4)
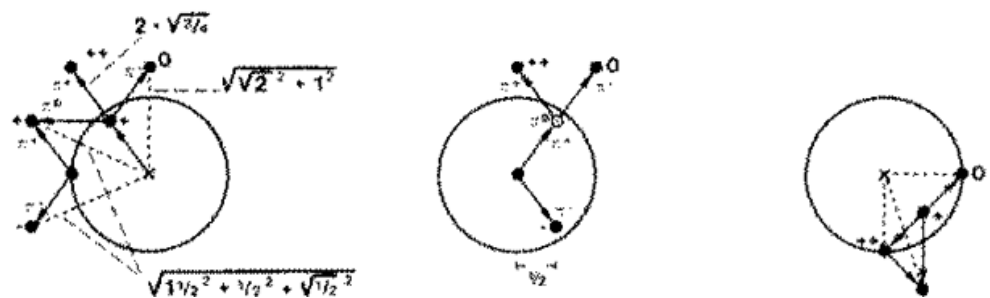

Fig. 4. The p-n, $\Lambda^{0}, \Sigma^{+, 0,-}$ and $\Delta^{++,+, 0,-}$ major semiaxis transformations, preserving volume and symmetry.

It is quite significant that the complete correspondence applies over the whole actual spectroscopy. Considering that all observed baryon particles and resonances in the $\Lambda, \Sigma, \Delta, \mathrm{N}, \Xi, \Omega$ and also full charm $[3-5,8,9,12-21]$ and bottom series are directly and reproducibly retrieved with just and no more than the actual states, channels, angular momentums, charge levels and precise mass numbers, and moreover in a faithful three-dimensional realization of the accepted eightfold way according to the original Lie prescriptions, the results are true and lasting. The reproduction of the charmed $\Lambda_{c}^{+}$(Fig. 7) serves to 
(E)

()
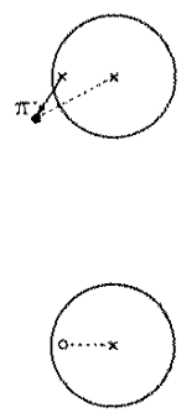
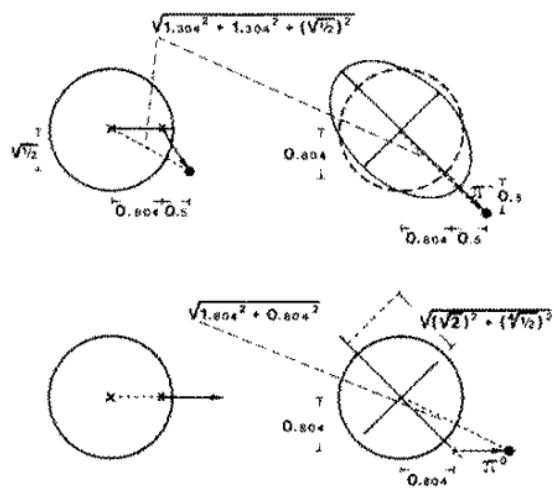

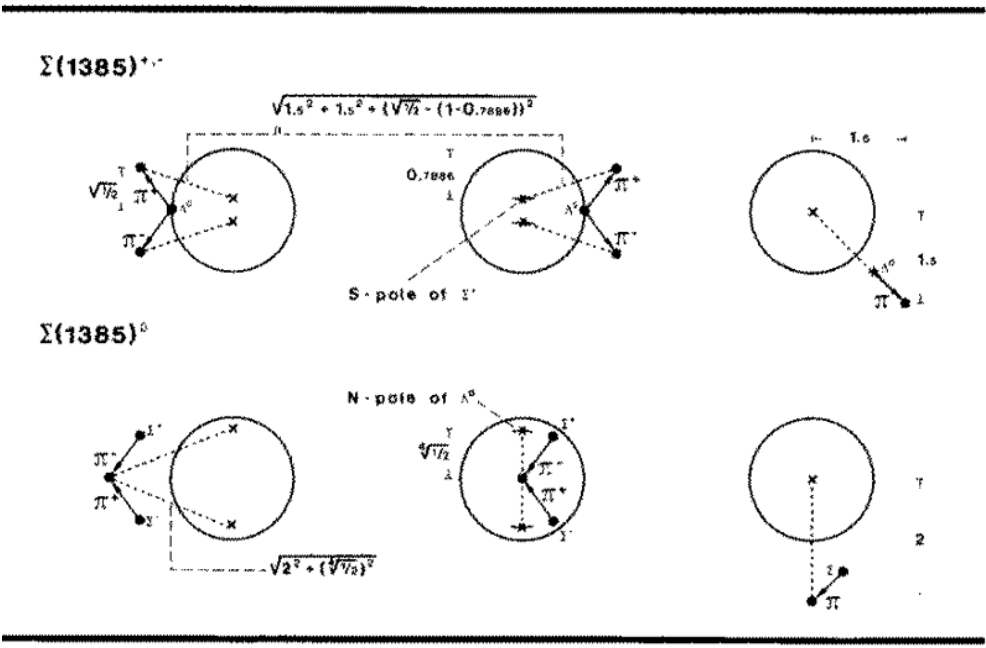

$\Lambda(1405)$
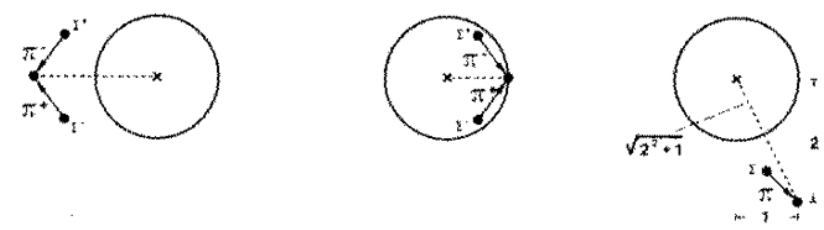

Fig. 5. The $\Xi^{0,-}, \Sigma(1385)^{+, 0,-}$, and $\Delta(1405)^{0}$ transformations.

substantiate this claim as further corroborated by all other charmed [14] and also bottom [8] hyperons in equal detail.

\subsection{The mesons}

Like the $\mathrm{A}_{2}$ diagram lattice by probabilistic permutations of root vector step sequences and semiaxis projections lodges all known baryons, also the mesons find a natural representation as differentials between the hadron states including themselves there. Their spatial framework is explicitly given by the 
Table 1

Lambda,sigma,delta,xi,sigma $\{1385\}$, lambda $\{1405\}$,xi $\{1530\}$ and omega hyperons, Masses (MeV) calculated according to formula, $938.27 \cdot 1 /$ minor semiaxis

\begin{tabular}{lllll}
\hline & Major semiaxis & Minor semiaxis & \multicolumn{2}{c}{ Mass } \\
\cline { 4 - 4 } & & & Calculated & Observed \\
\hline$\Lambda^{0}$ & $\sqrt{2}$ & $\sqrt[4]{\frac{1}{2}}$ & 1115.8 & 1115.6 \\
$\Sigma^{+, 0,-}$ & 1.60804 & 0.788591 & 1189.8 & $1189.4-1197$ \\
$\Delta^{++,+, 0,-}$ & $\sqrt{3}$ & $\sqrt[4]{\frac{1}{3}}$ & 1234.8 & $1230-1236$ \\
$\Xi^{0,-}$ & 1.975 & 0.7116 & 1318.5 & $1314.9-1321.3$ \\
$\Sigma(1385)^{+, 0,-}$ & $\sqrt{4.71}-\sqrt{4.75}$ & $0.679-0.678$ & $1382.2-1385$ & $1383-1386$ \\
$\Lambda(1405)^{0}$ & $\sqrt{5}$ & $\sqrt[4]{\frac{1}{5}}$ & 1403 & $1405 \pm 5$ \\
$\Xi(1530)^{0,-}$ & $\sqrt{7.06}$ & 0.6134778 & 1529.5 & $1528-1534$ \\
$\Omega^{-}$ & $2.505-2.51$ & $0.561-0.560^{*}$ & $1673.5-1677$ & $1672-1674$ \\
\hline
\end{tabular}

*Minor semiaxis changed in the transformation(c).

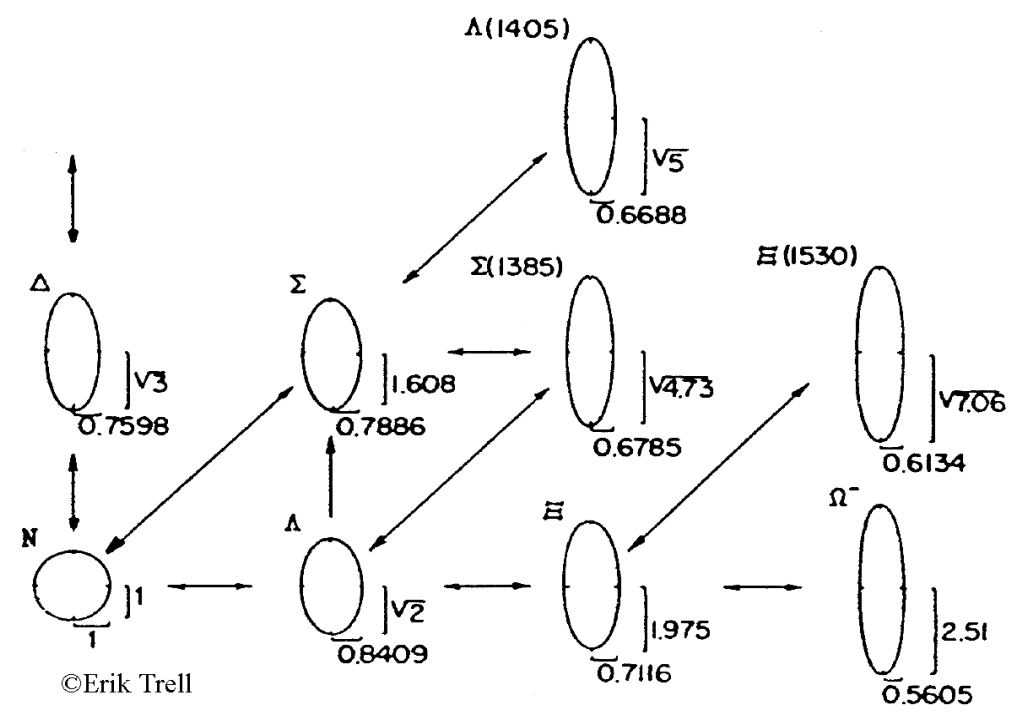

Fig. 6. Plane diagram of the appearance and channels of the basic eightfold way supermultiplets in the order they appear both in the real spectroscopy and the present faithful geometrical representation.

ordinary geometric representation of the established (symmetric) $\mathrm{SU}(2) \times \mathrm{U}(1)$ (antisymmetric) product group of the weak force $[5,12,13,20,21]$ and so they come out as polyhedral. Their channels are unit step ladders but the extensions of their outline mostly of other lengths and therefore, just as the baryon transformations unsustainable in the universal lattice, which has the structure of a space frame (Fig. 8), in whose build and transitions the mesons and leptons form the set of all possible bits and bytes and their assemblies in the kaleidoscopic build and reshuffling of the system. This becomes clearer in the following, starting with the neutral and charged pions which emerge as the first differential elements of the rectangular and hexagonal strands of the lattice, respectively. In Fig. 9 the neutral pion is shown, assuming the shape of a right circular cone segment with base area $1 / 4$ of the unit nucleon equatorial plane and length of spinning top generatrix $3^{1 / 2}$ so that the the mass expression of the enclosure of this ground neutral transformation step according to the aforementioned canonical group equation is $1 / 4 \times$ $938.27 \times 1 / 3^{1 / 2}=135.4 \mathrm{MeV}$ in comparison with the measured value of $135.0 \mathrm{MeV}$. 


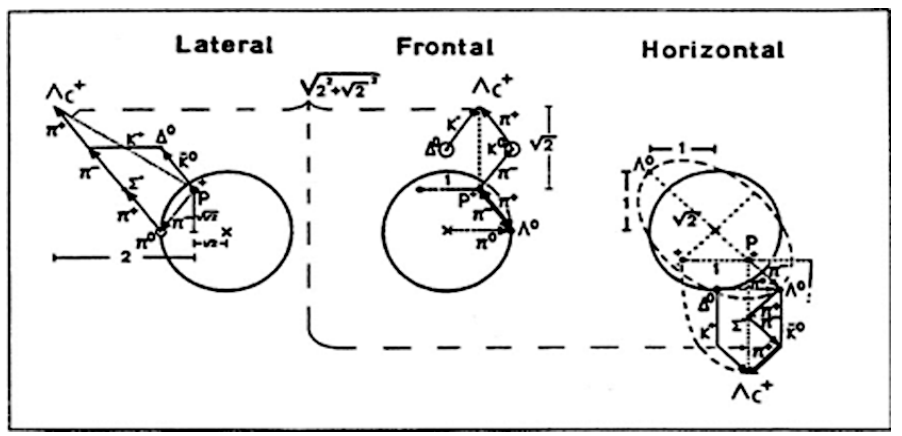

Fig. 7. Schematic plane view of he various $\Lambda_{\mathrm{c}}^{+}$projections in their simplest form. Only the major and minor semiaxis $\mathrm{b}_{2}$ projections are shown with equatorial plane of one (half) of the ellipsoidal transformations (from likewise outlined $\Lambda^{0}$ ) indicated in the horizontal projection. The channels are constituted by the unit $t$ isospin vector elements of the root space lattice, and it is seen that a multitude of them, e.g. $\mathrm{pK}^{-} \pi^{+}, \mathrm{pK}^{-} \pi^{+} \pi^{0}, \mathrm{pK}^{*}(892)^{-} \pi^{+}, \Lambda^{0} \pi^{+} \pi^{+} \pi^{-}, \Delta^{++} \mathrm{K}^{-}$and others, all equal to observed ones, can lead to the new major semiaxis end-point(s), which in all varieties outline a major semiaxis length of $\left[2^{2}+\left(2^{1 / 2}\right)^{2}\right]^{1 / 2}=6^{1 / 2}$. In a symmetrical transformation the minor semiaxis $(1 / 6)^{1 / 4}$ gives rise to the $\mathrm{N}(1440)$ resonance [5], while the exotic charmed transformation event results from incorporating one minor semiaxis $=1$, so that the changed minor semiaxis length is $1 /(6)^{1 / 2}$ with resulting mass $6^{1 / 2} \times 938.27 \mathrm{MeV}=2.30 \mathrm{GeV}$ compared with measured mass $=2.29 \mathrm{MeV}$ The same occasional incorporation of one minor semiaxis $=1$ leads to exact matches also in remaining charmed baryons [14].

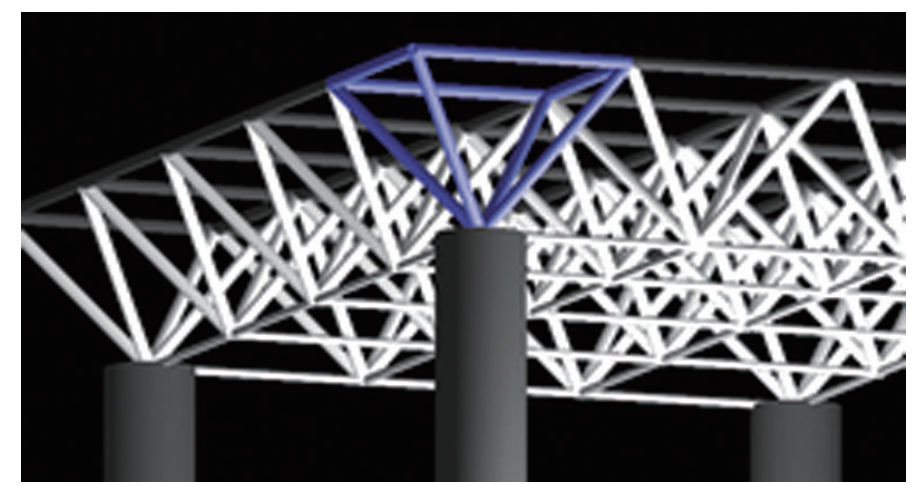

Fig. 8. (adapted from Wikipedia) Simplified space frame roof with half-octahedron highlighted. A space frame or space structure is a truss-like, lightweight rigid structure whose geometry is most often based on the Platonic solids. The simplest form is a horizontal slab of interlocking square pyramids. A stronger purer form is composed of interlocking tetrahedral and octahedral crosspieces in which all the struts have unit length. More technically this is referred to as an isotropic vector matrix or in a single unit width an octet truss. More complex variations change the lengths of the struts to curve the overall structure or may incorporate other geometrical shapes.

The charged pion (Fig. 10) comes equally swift out in the faithful transition lattice as an oblique circular cone, wrapped between state transformations in the hexagonal half-part since happening between state transformations in the hexagonal lattice moiety where it occupies a base area of 1/6th of the proton equatorial plane with average generatrix length of $(5 / 4)^{1 / 2}$ so that the mass number is $1 / 6 \times 938.27 \times$ $1 /(5 / 4)^{1 / 2} \mathrm{MeV}=139.9 \mathrm{MeV}$ versus the observed $139.6 \mathrm{MeV}$.

Again is noted an unprecedented identity between reality and replication also when it comes to exhaustiveness; no other varieties at the respective levels occurring in any of the systems. And the same correspondences according to the unmistakable scientific directions continue in all other mesons $[5,12-$ 21] as exemplified in the next basic states (Fig. 11); since long then by any probability testing and other rigorous authenticity criteria surpassing the slightest possibility of a chance coincidence. 


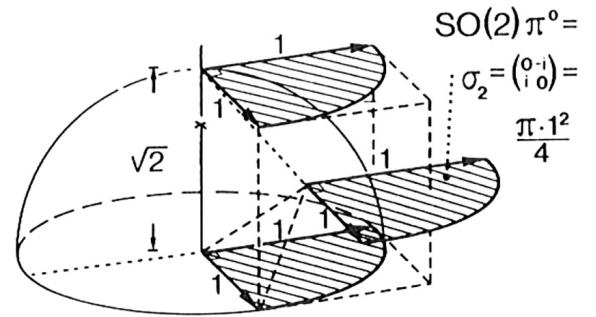

(a)

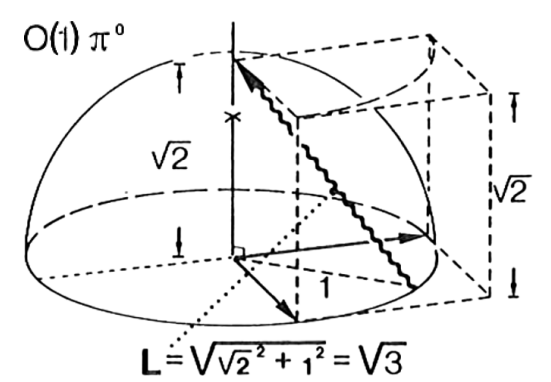

(b)

Fig. 9. (a) A neutral $t$ isospin root vector is inclinated $90^{\circ}$ to adjacent neutral isovector doublets in the lattice. Between each other they form a circle sector amounting to one quarter of the equatorial plane of the proton. (b) The distance to the next neutral $\mathrm{SO}(2)$ counterpart of the $\mathrm{SU}(2)_{\mathrm{wk}}$ isospin planes in the transition lattice is $3^{1 / 2}$.

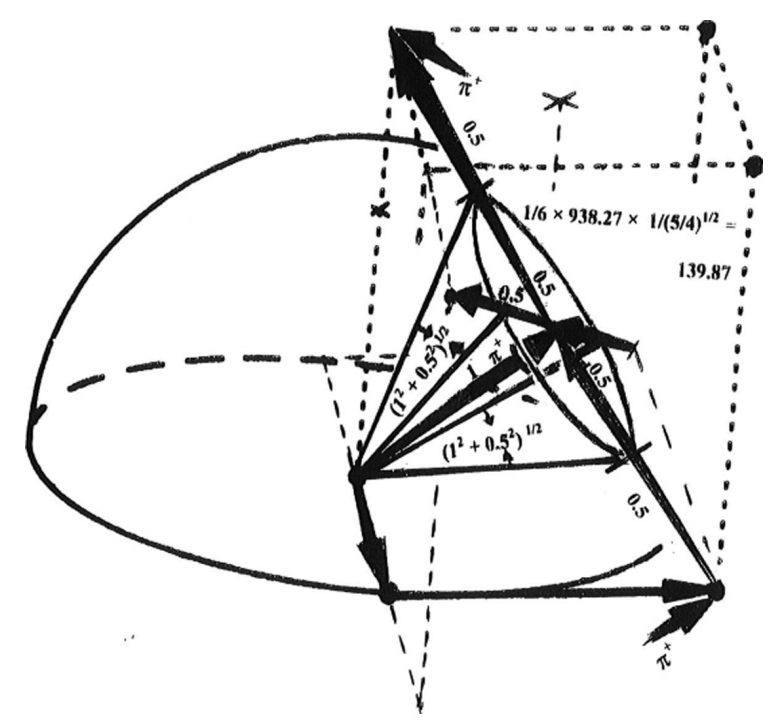

Fig. 10. The charged pion takes place in the lattice as the primary differential enclosure of single-step transfor-mations between charged hadron states, covering a conical base area $1 / 6^{\text {th }}$ of the size of the proton equatorial plane centered along an $180^{\circ}$ (muonic) root vector sequence and with an (average) generatrix length of $5 / 4^{1 / 2}$.

It is striking and convincing that polyhedral root space elements, both differential and equilateral, are so in double sense straightforwardly involved and that it is possible to exactly and exhaustively match the observed elementary particle spectroscopy by classical solid metamorphoses. Table 2 exemplifies the mass number computations in the shown states according to the $\mathrm{SU}(2) \times 1 / \mathrm{U}(1)$ Lie algebra relation of the weak interactions, and the parallelism in every regard; also chargewise and channelwise is manifest.

And it continues in the entire plethora of mesonic differentials and transformations over the whole spectrum of towering hadrons up to charmed (Fig. 12) and bottom and even top flavours [8,14,20,21].

\section{The leptons}

The mesons have been faithfully replicated here as spinning residual volumes comprising the additive partial differentials of the involved transformations between all hadron states, very much like bubbles 
Table 2

Basic mesons calculated and observed mass numbers $(\mathrm{MeV})$

\begin{tabular}{llll}
\hline$\pi^{0}$ & $1 / 4 \times 938.27 \times 1 / 3^{1 / 2}$ & 135.4 & 135.0 \\
$\pi^{ \pm}$ & $1 / 6 \times 938.27 \times 1 /(5 / 4)^{1 / 2}$ & 139.9 & 139.6 \\
$\mathrm{~K}^{ \pm}$ & $938.27 / 4 \times 1 / 3^{1 / 2}+938.27 / 4 \times 1 / 3^{1 / 2}+938.27 / 6 \times 1 / 2^{1 / 2}$ & 492.0 & 492.7 \\
$\mathrm{~K}_{\mathrm{S}}^{0}$ & $938.27 /\left(4 \times 1 / 2^{1 / 2}\right)+938.27 /\left(8 \times 1 / 2^{1 / 2}\right)$ & 497.6 & 497.67 \\
$\mathrm{~K}_{\mathrm{L}}^{0}$ & $938.27 /\left(8 \times 1 / 2^{1 / 2}\right)+938.27 /\left(8 \times 1 / 2^{1 / 2}\right)+938.27 /\left(8 \times 1 / 2^{1 / 2}\right)$ & 497.6 & 497.67 \\
$\eta$ & $938.27 / 6+938.27 / 6+938.27 / 4$ & 547.33 & $548.8 \pm 0.6$ \\
$\rho(770)$ & $\left(938.27 / 1 / 2^{1 / 2}\right) /(3 / 4)^{1 / 2}$ or $\left(938.27 \times 2^{-2}\right) / 3^{1 / 2}$ & 766.1 & $768.3 \pm 0.5$ \\
$\omega(783)$ & $938.27 / 4+938.27 / 4+938.27 / 6+938.27 / 6$ & 781.9 & $781.95 \pm 0.14$ \\
\hline
\end{tabular}

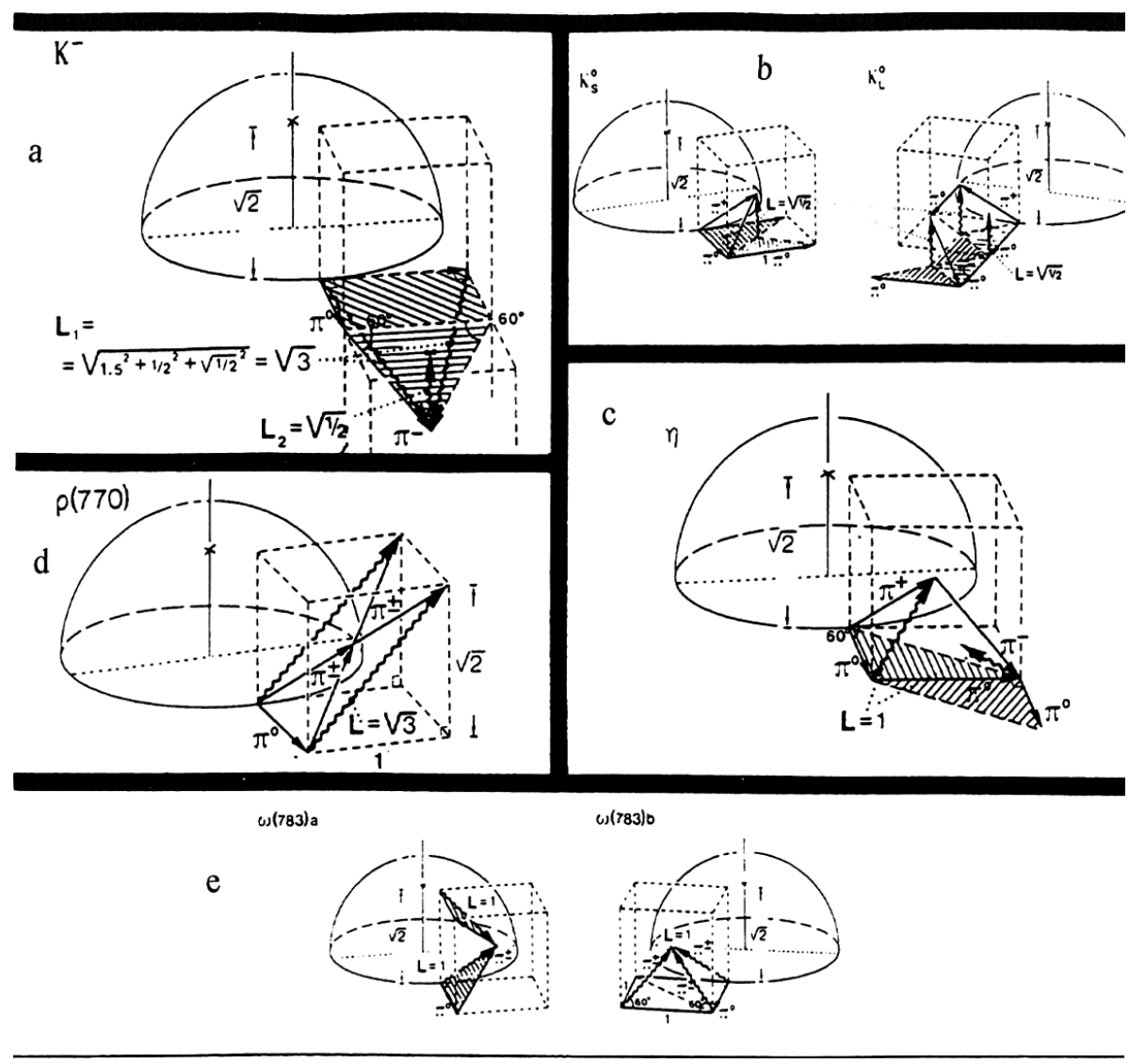

Fig. 11. Chart of (a) charged kaon; (b) $\mathrm{K}^{0}$ short and $\mathrm{K}^{0}$ long; (c) $\eta$; (d) $\rho$ (770); (e) two forms of $\omega$ (783).

bursting to smaller bubbles before they end up in a spray of linear jets. The latter are the leptons, which, apart from the further decaying tau and muon, are the irreducible extra-nucleon vector elements of the infinitesimal world wide web that we are literally intertwined with.

Paradoxically, despite their plain one-dimensionality and limited number of states, the leptons stand forth as the perhaps most elusive of the elementary particles. With the exception of tau, their antisymmetric Lie algebra is $\mathrm{U}(1)[5,12,13,20,21]$, whose geometric isomorphism is the ordinary real line, the composed length of which may accordingly vary. However, already in the existing wave model it is at the limes level put together by infinitesimal derivatives which are straight unit bits meaning that, inner- 


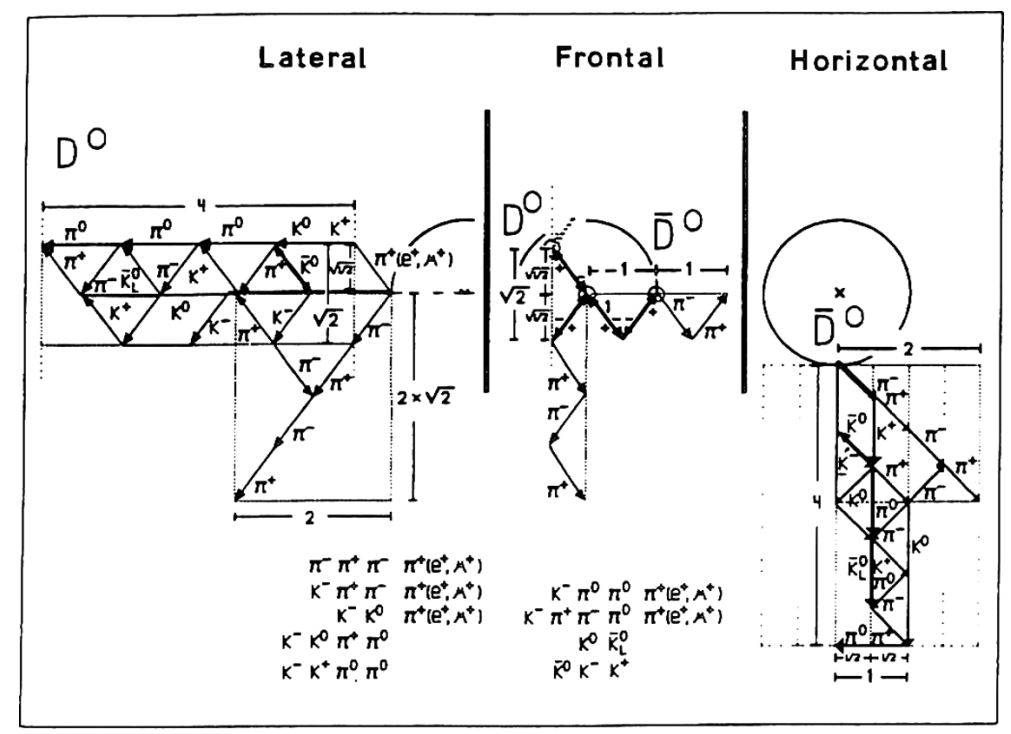

Fig. 12. The identity between reality and animation exemplified by the charmed $\mathrm{D}^{0}$ and its $\overline{\mathrm{D}^{0}}$ antiparticle equally many levels out in the lattice as in practice [14]. All channels can be retrieved (only a sample shown here), spanning SO(2) planes along the vertical axis tentatively assigned to $\mathrm{D}^{0}$, and the horizontal axis assigned to its antiparticle. The area in the first case is $\pi \times 4 / 2 \times 2^{1 / 2}$ or $\pi \times 2 / 2 \times 2\left(2^{1 / 2}\right) / 2$; both $2^{1 / 2}$ larger than Proton equatorial plane. With distance to next $\mathrm{D}^{0}=1 / 2^{1 / 2}$, the mass number is $2^{1 / 2} \times 1 /\left(1 / 2^{1 / 2}\right) \times 0,93827 \mathrm{GeV} \approx 1.88$ vs the recorded $\approx 1.86 \mathrm{GeV}$. In the antiparticle the spheroidal area equals the Proton and with interdistance $1 / 2$ gives the same result.

most, the lepton scalar world function emerges as digital. So is likewise the case in the $\mathrm{A}_{2}$ lattice. The infinitesimal straight line digit, or 'pixel' is immediately embodied in the uniform, sole ingredient unit root vector element of either neutral or charged inclination, whose iteration is everything that constitutes the lattice and the hence eigen-spacefilling geodesics there. That close matches with the leptons are indeed manifest in it is therefore not surprising in regard of the regular solids' (slightly oxymoronic) 'unique universality', but nonetheless truly remarkable. In fact, the leptons weave the extra-nucleon world, and with such extreme simplicity that is has been overlooked for that very reason.

Starting with the particulate leptons, there are two principal ways of connecting the ultrasharp charged root vectors of same sign, here exemplified by the positive muon and the positron, viz., in the first case, by $90-180^{\circ}$ turns (Figs 13 (a)-(c)), and, in the second case, $60-120^{\circ}$ turns (Figs 13(d),(e)).

In the figure, showing that the scheme has high relevance also in the antimatter context, the positron is traced by linking the positively inclined root vectors (electron mirrored when the negative are connected). The $90-180^{\circ}$ alternatives (Figs 13(a)-(c)) form plane or helical orbits from, over and outside of the nucleon surface with a unit scale length in all varieties of $\left(2 \pi \times 2^{1 / 2}\right)$ or $\left(2 \pi \times 2 \times 1 / 2^{1 / 2}\right)$ and resulting mass number $1 /\left(2 \pi \times 2^{1 / 2}\right) \times 938.27=1 /\left(2 \pi \times 2 \times 1 / 2^{1 / 2}\right) \times 938.27=105.59 \mathrm{MeV}$ in comparison with the measured muon ${ }^{ \pm}$mass of $105.66 \mathrm{MeV}$. In the second alternative, a three-winged orbit can be tied together (Figs 15(d),(e)) and leads out of the nucleon surface, so that it is natural to associate it with the positron/electron trajectory. The circular orbital length of the ground rosette is easy to calculate as $3 \times\left(2 \pi \times 1 / 2^{1 / 2}\right)$ in unit gauge, but it is well known that one has to multiply with the fine structure constant, $137.035986 . .$. , to obtain the first, in this case 'Mercedes star' threepronged circumference, so that the ground state positron/(mirror)electron mass number comes out as $1 /\left(137.035986 \times 3 \times 2 \pi \times 1 / 2^{1 / 2}\right) \times 938.27=0.514 \mathrm{MeV}$ in comparison with the recorded $0.511 \mathrm{MeV}$. 


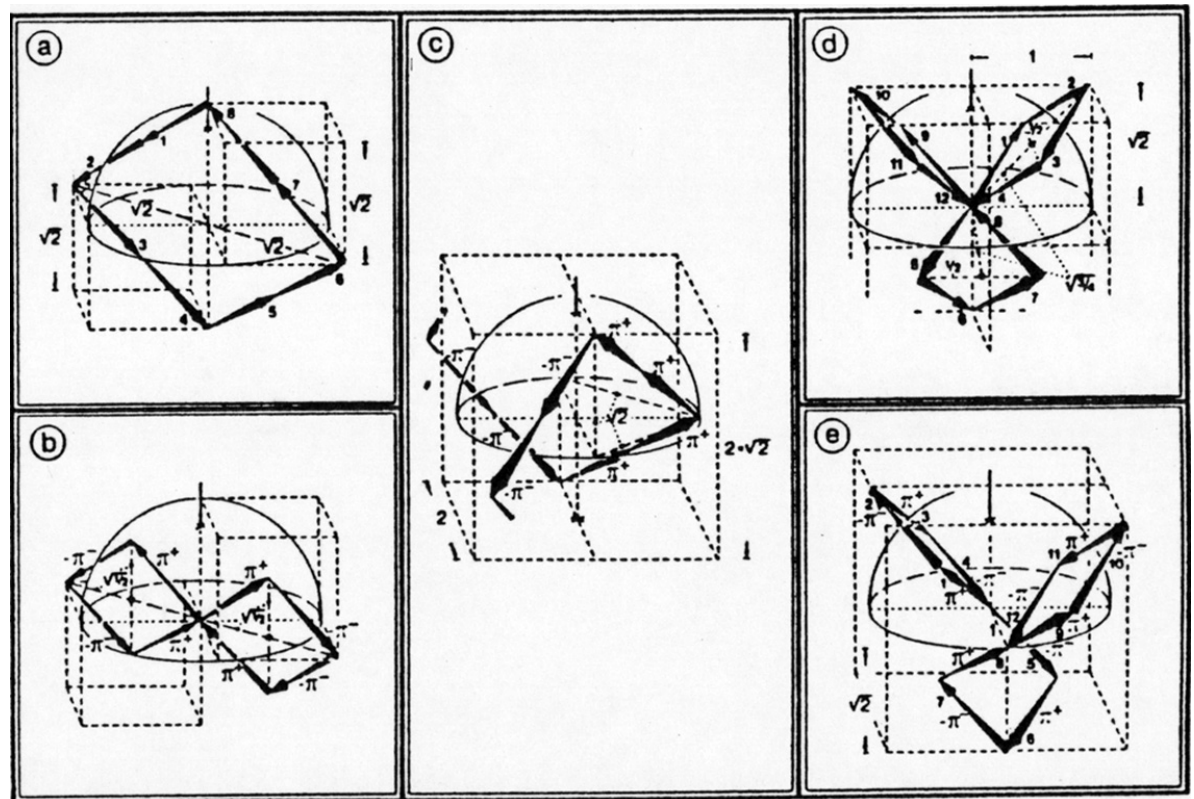

Fig. 13. Cores of (in this case positively charged) lepton geodesics over nucleon surface.

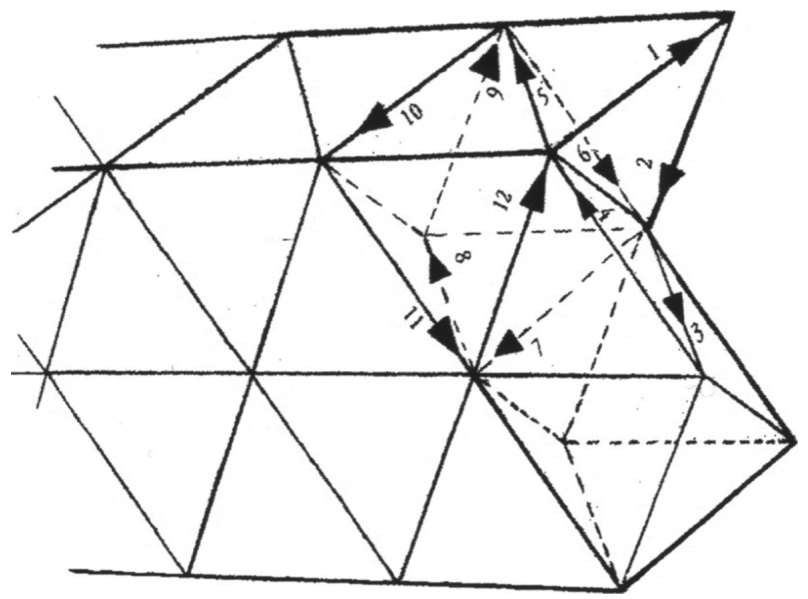

Fig. 14. The figure shows a continuous outlining of the spacefilling one octahedron/two-tetrahedrons root vector lattice coming back to the origin and thus a closed loop. Only the charged root vectors are involved.

However, there are problems with the orbital model, for instance, in terms of the then alien, empty region under and between its rings. For consistency, a truly spacefilling distribution is wanted. Being a sequence of unit steps there would be no difference in principle in relation to the orbital model, which, as mentioned, is also composed of iterated infinitesimal straight line intervals. And there exists such possibilities which can be patched together to larger structures in a hierarchically periodic fashion just as in modern nanotechnological self-assembly [20,21]. One of them is the truncated octahedron which is a composite space-filling Archimedean solid that already Kepler saw as fundamentally engaged in the cosmographical architecture. [Ib.] The truncated octahedron distribution of a full positron/electron turn 


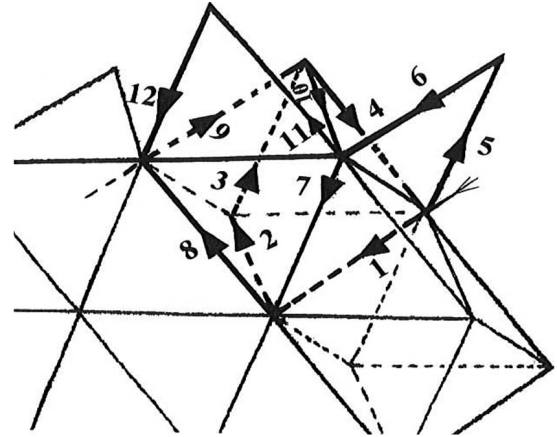

(a)

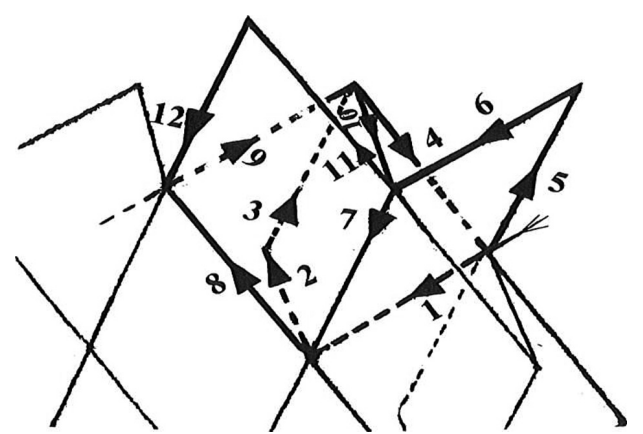

(b)

Fig. 15. Another, open loop, allowing a spacefilling continuous, e.g. helical path by the charged root vectors alone. Each (quarter) turn consists of six two-side corners which are present also in the ground cube.

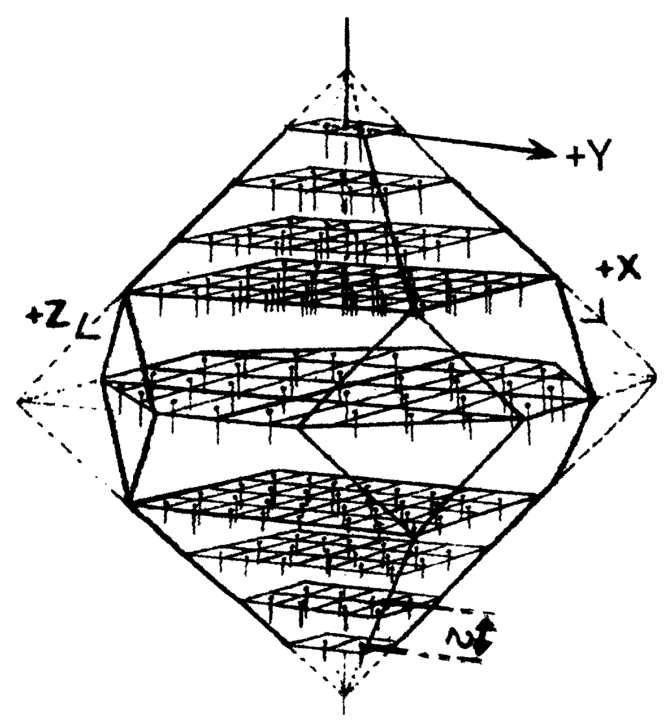

Fig. 16. Truncated octahedron distribution of 152 electron twelve-step nodes (their triple coils just indicated as rods) in two vertically joined Cartesian segments. The corresponding Bohr orbital shells are shown to the left.

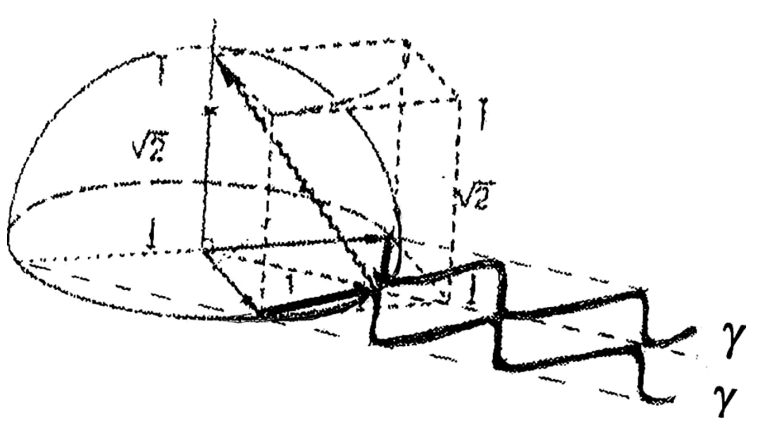

Fig. 17. Example, in the rotating top differential neutral pion, how photons are generated when root vectors in e.g. particle decay or Brehms-strahlung bendings within same charge plane snap back to their space axes setting up a zig-zag ripple between them of infinite length, thus zero mass and amplitude/frequency also determinable.

may follow from the only spacefilling sequence of the charged root vector lattice, namely (Figs 2(d),3), a twelve-step, two-tetrahedrons/one octahedron triple coil node, or 'rosette', generated by the local quantum fluctuation distributing its twelve, equally many as the cube, sides into the coherence of the spherical root vector lattice, as shown below in one variety of a twelve-step loop returning to the origin as a veritable casting-on stitch of the web (Fig. 14).

Figure 15 demonstrates another alternative with traits of the harmonic oscillator [10, p. 551], where the tetrahedral rosette wings like a spinning cube turn $90^{\circ}$ around the corner enabling a variety of continuous patterns and also (Fig. 15(b)) that it consists only of (equally) charged root vector steps.

Figure 16 sketches how 152 such 12-step Electron rosettes in Bohr orbital layering may fill, by in all 1852 charged root vector steps (half) a truncated octahedron, so that the inertia/mass is $1 / 1852 \times 938.27$ $=0.514 \mathrm{MeV}$ in comparison with measured $0.511 \mathrm{MeV}$. Before coming back to this projected primary 
electron cloud of the Hydrogen ion and its onward atomic and Periodic Table expansions, the remaining leptons; the photon, and the muon, electron/positron and tau neutrinos and anti-neutrinos will be briefly considered. They occur, in the lattice as in reality, as one-dimensional differentials, e.g., when a larger transition portion there such as the neutral pion decays like an imploding bubble into two $\gamma$.s (Fig. 17). Common to all photons, they get a sinusoidal trajectory of wavelength determined by the inclination of the zig-zagging iteration of their difference root vector. Regarding the neutrinos/anti-neutrinos, Fig. 18 demonstrates how the charged pion regularly decays into a muon and a muon neutrino, whose forward movement vector, abandoned by the muon, continues in an endless and hence massless straight course which will pass without interaction throughout the entire global latticeunless head-on intercepting with an element there.

And this extensive correspondence of nothing more, nothing less and all the same persists in the metastable muon, which outside of the nucleon is destined to bend its $90-180^{\circ}$ surface track into the extranuclear lattice course of the electron/positron thus setting up a muon neutrino and electron antineutrino (Fig. 19). Even the tau is easy to identify and reproduce as a direct constituent and event in the root space. As a lepton, tau is a bit odd because it decays also weakly, governed by the mesonic (S)U(2) symmetry group. It is produced inter alia by high-energy electron-positron collisions and it is indeed possible to extract matching SU(2) planes, to the area of which the mass number should be directly proportional in relation to that of the proton equatorial plane $(\pi)$, both in the intra-nucleon and extranucleon electron/positron modules. In the former, the 'Mercedes star' (Fig. 13d) as well as the helical path (Fig. 13e) contains three square/rhombic planes of unit size and the same applies to the external triple-coil singlet 'rosettes' (Figs 14-15). In any casethe added area is thus $3 \times 1$,whichin a tangential encounter between an electron an a positron could be uncoiledfrom each of these so that the total mass number equation will be $2 \times 3 / \pi \times 0,93827 \approx 1.79 \mathrm{GeV}$. This compares very well with the recorded tau mass of $1,78 \mathrm{GeV}$ and also in other respects, like production and decay modes, the correspondences are extensive.

Table 3 summarizes the results in the leptons accounted for here.

Table 3

Basic lepton calculated and observed mass numbers

\begin{tabular}{lllll}
\hline$\tau^{ \pm}$ & $2 \times 3 / \pi \times(0.93827)$ & 1.79 & 1.78 & $\mathrm{GeV}$ \\
$\mu^{ \pm}$ & $1 /\left(2 \pi \times 2^{1 / 2}\right) \times 938.27$ or $1 /\left(2 \pi \times 2 \times 1 / 2^{1 / 2}\right) \times 938.27$ & 105.6 & 105.7 & $\mathrm{MeV}$ \\
$\varepsilon_{\text {orbital }}^{ \pm}$ & $1 /\left(137.035986 \times 6 \pi \times 1 / 2^{1 / 2}\right) \times 938.27$ & 0.514 & 0.511 & $\mathrm{MeV}$ \\
$\varepsilon_{\text {solid }}^{ \pm}$ & $1 /(152 \times 12) \times 938.27$ & 0.514 & 0.511 & $\mathrm{MeV}$ \\
$\gamma$ & $1 / \infty \times 938.27$ & 0 & $0\left(<3 \times 10^{-33}\right)$ & - \\
$\nu, \nu_{\tau \mu \varepsilon}$ & $1 / \infty \times 938.27$ & 0 & $0(<17-35)$ & - \\
\hline
\end{tabular}

\section{Real form in real space}

As a matter of fact as well as, in that sense, fact of matter, reproducible descriptive results have been disclosed here that unmistakably relate and correspond to canonical scientific theories and observations, and for the first time complement them with a detailed and exact ordinary structural modelling and computational method. However, it may be ventured that they, like these "best attempts at the ultimate explanation of matter ... suffer from a fundamental weakness", since they might seem to be mounted ad hoc "in a spacetime whose shape has been chosen from the beginning, as if they were actors on a previously constructed stage", whereas "a truly fundamental theory would build the stage itself" [2]. 


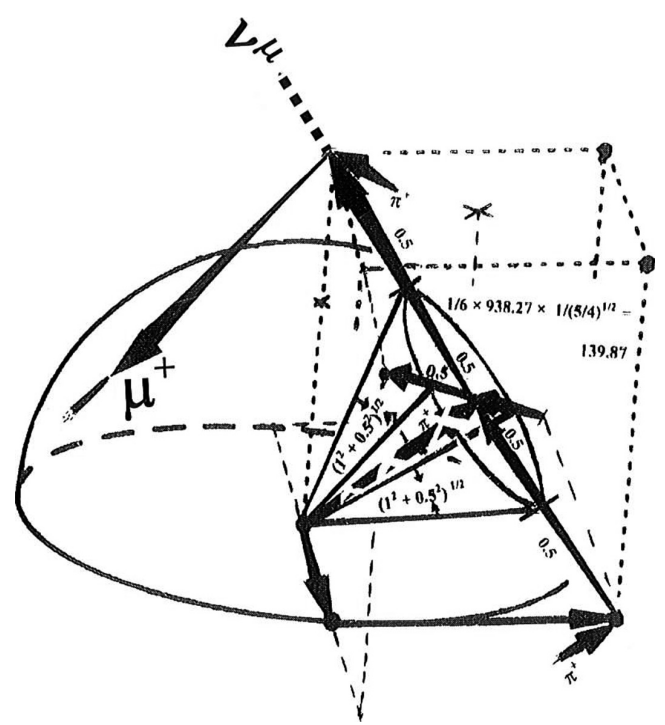

Fig. 18. As indicated in the axis and demonstrated in the neighboring site, the charged pion decays by a muon (compare Fig. 13(a)) and an ongoing $\nu^{\mu}$ momentum vector.

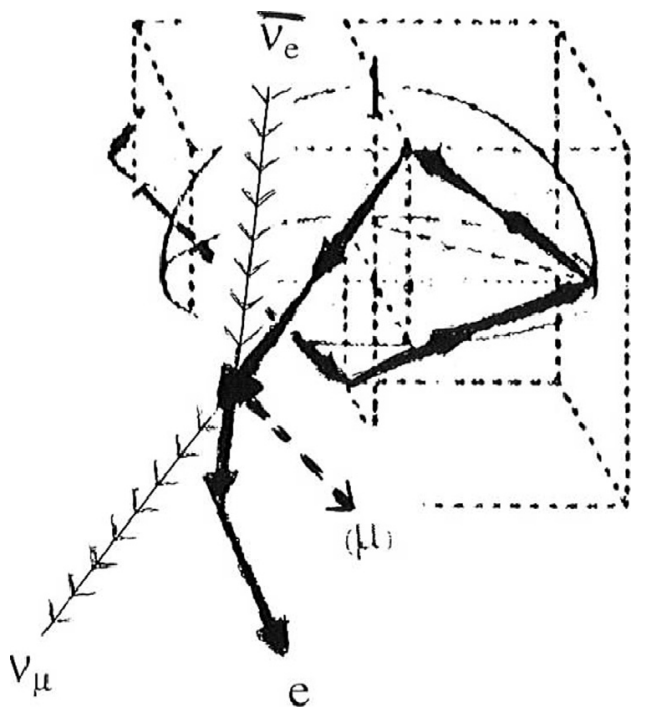

Fig. 19. Example (compare Fig. 13(c)) how a transition of, in this case, the muon into the electron geodesics leaves straight forward Bra/backward Ket momentum vectors corresponding to muon neutrino and electron antineutrino, respectively, both endlessly extending over successive space lattice intervals and hence of $1 / \infty=$ zero mass and nil amplitude.

But, in a slightly tautological way this is rather the corollary, that the shape of real space is chosen from the beginning because it is the true shape of real space and it thus builds its own stage itself due to its actual constitutional reality. And this is also the ancient both intuitive and ontological understanding of it not as alien and strange but akin, even parent to and fabric and in a double meaning incubator of matter. For instance, Euclid's own formulation of a fundamentally flat three-dimensional Cosmos and the geometry and processes therein "was originally based on watching how people built...of cubes with sides of standard length required to fill a solid space" [22]: the space is filled with cubes which are in turn the parts of the space, or, in modern terminology its self-assembling eigen-elements and hence implicitly analytic since every piece can only occupy its own one and only place.

This insight is getting even more significant in the context of the regular solids, of which the cube was assigned to the composition of the earth which in a geocentric conception translates to the spatial structure as well. But even more significantly, the ground element of the cube is the unit equilateral straight line, defining and setting its own conditions as first explicitly surveyed by Ptolemy, who in his book On Distance (150 AC) wrote: "Draw three mutually perpendicular lines. Try to draw another line perpendicular to all of these lines. It is impossible. The fourth perpendicular line is entirely without measure" [20]. Figure 20 summarizes his formalization of the old idea that space and matter are two sides of each other and made by the same stuff, which has also been revived in digital computer terms [23], corresponding to the screen and processor parts of the integrated system, joined and reinforcing each other in all computational and realization operations. [Ib.] The holographic 'canvas' [24] of the real three-dimensional Cosmos with its cubical crystal [25] composition follows directly from the fact that its building element, the straight line, can span no more than three linearly independent space axes. (Fig. 20).

Also Aristotle (384-322 BC) thought that the world is three-dimensional and that the formations in it are timeless, their simultaneous strands interpunctated by successive smallest steps which both as 


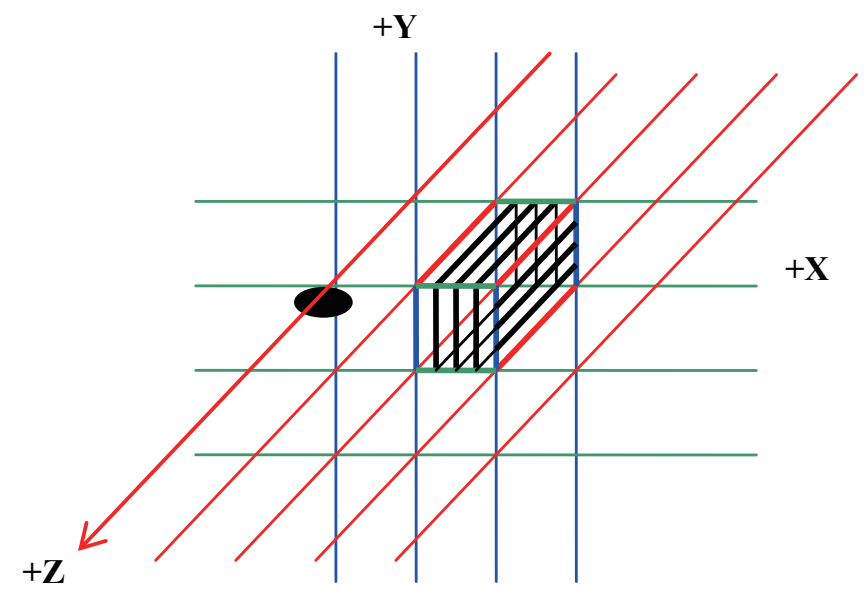

Fig. 20. Say, that there comes a straight line out from one's closed eyes leaving in the forward direction $(+\mathbf{Z})$. Then it must also endlessly extend towards one from behind, and there must be such lines infinitesimally tight over all the void's reach, because a linearly independent such axis can also come from below and rise up $(+\mathbf{Y})$, or from the one side and leave on the other $(+\mathbf{X})$, all of them together thereby spanning the endless Cartesian co-ordinate system and between them enclosing the infinitesimal cubical eigenvector bits of the matrix.

cause and effect mark the moments of the sequence as well as of the interaction cone of the advancing front of apparent now. At any scale, the consequential Cartesian eigen-coordinate system (Fig. 21) and the realizations within it are therefore both 'Gödel-immunized' and 'Popper-ratified' since defined and constituted and sensu strictu falsified solely by themselves.

The virtual Minecraft-like cubical constitution of the cosmic screen holds quite interesting pure mathematical powers, e.g. scale-independent on-line mapping of the complete Diophantine equation space and instant solutions of Fermat's Last Theorem and Beal's conjecture [20,21,26-32], but has no dynamic function or action per se in its absolutely still, absolute zero deep-frozen state. However, taken down to the smallest size where the Ptolemian crisscross lines come so close that they touch, the rendered global matrix of minuscule cubical eigenvector pixel cells, or cuBits ${ }^{\circledR}$, contains all the infinitesimal unit length vectors it is enclosed by, and therefore serves as the equally concrete as abstract substrate and 'tool box' for a possible dynamic outlining of this root space element in automatically the same scale and amount. Everything that there is to use then is still the unit straight line, and must in order not to be annihilated by inner dissonances happen by and as a systematic breaking up and redistribution of the sides of the cubes, lending this unique vector quantity for the necessarily commensurately ordered and uniform and space-filling process, of which there is only one possibility, namely a phase transition to a composite tetrahedron/octahedron Platonic solid organization. (Figs 14 and 15). As indicated in Figs 2 and 3, this is precisely the $\mathrm{A}_{2}$ root vector diagram and lattice and can be described as a hybridization of the unit sphere within a Cartesian Lie neighbourhood of eight unit cuBits (Fig. 22), where the actual phase transition originates in any of them, which is relevant in the present context of anti-matter and inverses, too, since these can be lodged in the diagonal segments. In casual latter-day parlance one might refer to the contraption as a two-stroke phase motor, but it is not just heuristically contrived to fit but has a deep philosophical and epistemological underpinning. As cited from [20]: "Since ancient time a profound insight in relation to the straight and round forms is that they are absolutely endless, yet distinct and irreconcilable over a gap of limes (last decimal of) $\pi$. They are alternative versions of one cardinal realization, and this statutory dichotomy like two juxtaposed conduction plates generates a potential fall between the respective, maximally dilated versus maximally contracted infinite strands. One may here 


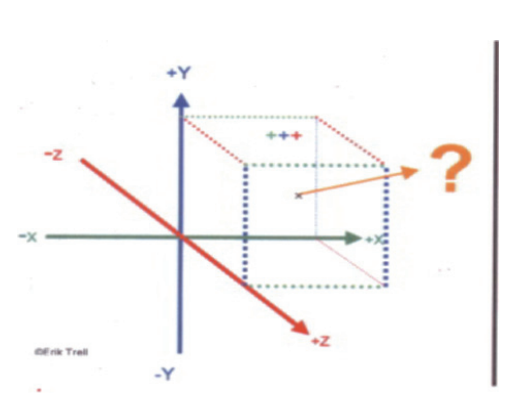

a)

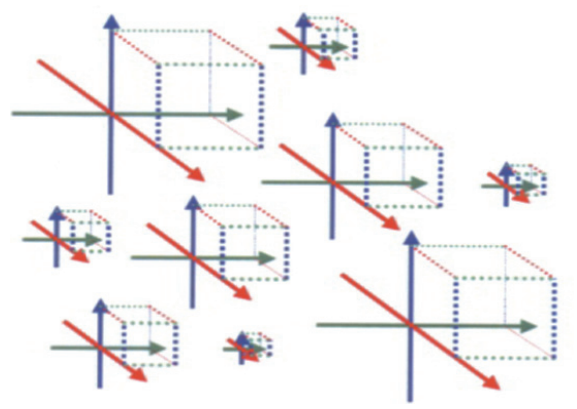

b)

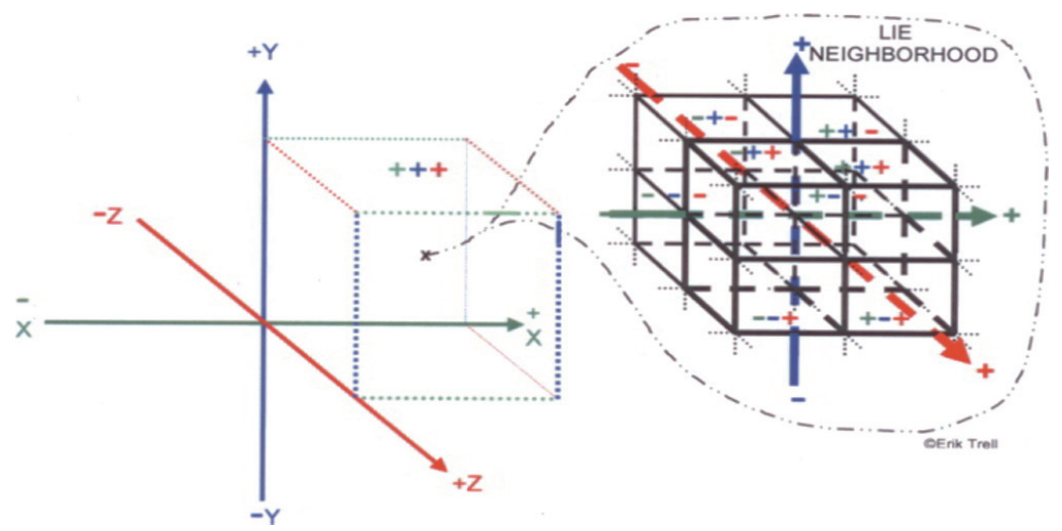

c)

Fig. 21. a) The Cartesian coordinate system spans the three-dimensional Euclidean space in eight cubical segments. What is the constitution of a local part (?) in any of them? b) Regardless of size it retains the Cartesian representation. c) Hence, the smallest composite space portion is a Lie neighbour-hood of eight indivisible ground unit CuBits.

quote Aristotle (384-322 BC): 'everything that comes to be comes into being from its contrary ... and passes away likewise ... by the action of the contrary into the contrary' and 'if there is a contrary to circular ... a straight line must be recognized as having the best claim to that name"' [20,21]. Furthermore, Marius Sophus Lie himself in his doctoral thesis "Over en Classe Geometriske Transformationer" stated that they were essentially "between the Plücker line geometry and a geometry whose elements are the space's spheres" $[6,7]$.

The cubical and spherical root space lattices coincide along the equatorial Cartesian coordinate axes (Fig. 2) but are otherwise disjoint so that they exist together as two reciprocal modes of arranging an equal amount of identical root vector elements simultaneously. However, there is a principal difference between the cubical and the spherical symmetry lattice in that the former folds onto the cuBit sides whereas the latter spans a vector from the centre towards the inside of the unit sphere from which it is free to fan out along opposite charge sign to preserve the net neutrality (Figs 1-3). An important consequence of this reflective root vector coset decomposition on either side of the nucleon surface is the parting into the internal baryon and external meson and lepton geodesics with their synchronization and exchange by mutual spacefilling and the electroweak and strong interactions. Figure 23 recapitulazes the organization of the structure that is then in stock for the electron/positron distribution, and it is seen that it is consisting of the diagonal vector elements alone and formed into octahedron sides with tetrahedral niches in-between so as to completely fill the space. And even more compelling, and with 


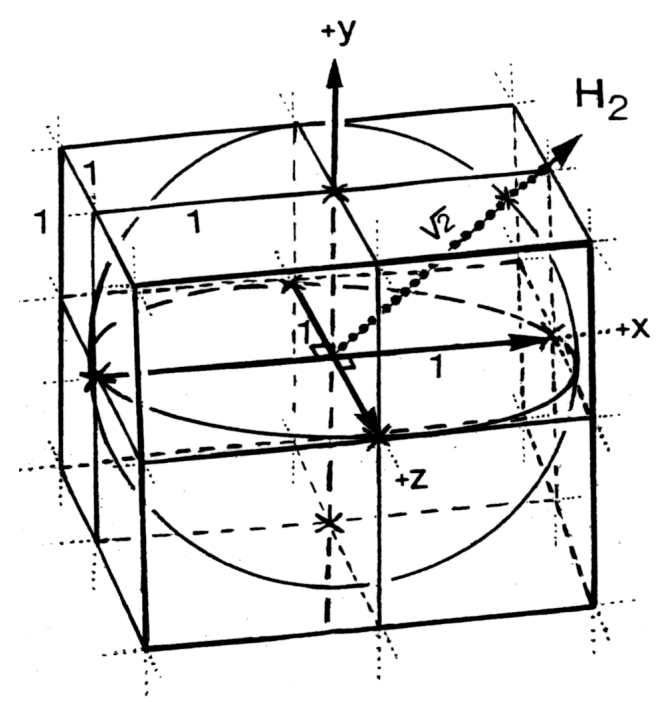

Fig. 22. The hybridization of the unit sphere within the cubical Lie neighbourhood sets up an interstice in the universal iteration of which the basic tetra- and octahedral regular solid phase transition is immanent.

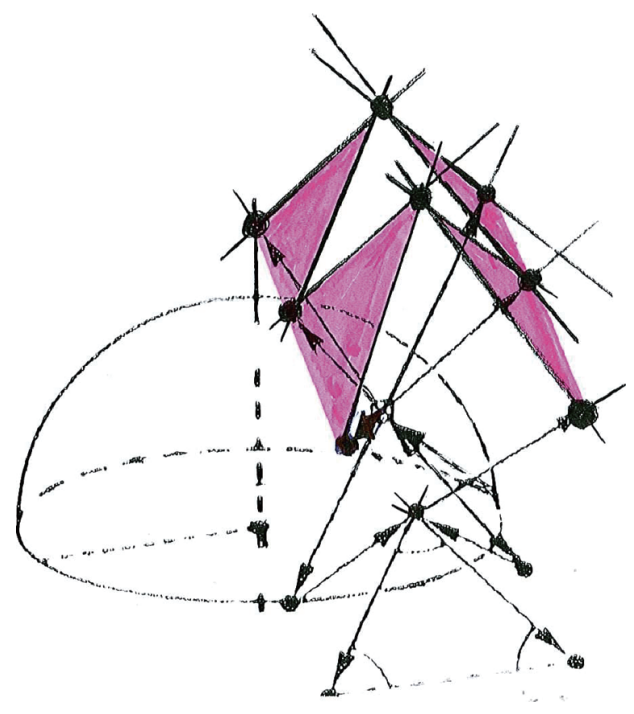

Fig. 23. A dissection of a portion in the first and second extranucleon layer of the charged $t$ isospin root vector lattice, showing that it connects as octahedron sides without involvement of any neutral root vector elements.

reference to Figs 13-15, Fig. 24 demonstrates that the outlining of the octahedron/tetrahedron complexes represented as rods in Fig. 16, can be in a periodically continuous sequence going into space-filling Archimedean solid or other geometrical domains which can then template hierarchically expanding selfaggregation of its self-similar motif to atoms and larger structures direct into the approaching frontline of advancing nanotechnology [20,21]. Both are and cannot be more than three-dimensional, meaning, firstly, that an actual time dimension does not exist $[33,34]$ but in the Aristotelian sense is a simultaneous interpunctation of the events; secondly, that these occupy their domains at once and forever; thirdly, that the elementary particles and atoms must then be isometrically dispersed from the outset so that, fourthly, the instant inflation [1] throughout space of the collective Big Bang is offered for free.

\section{Stacking the electron cloud in periodic system expansion}

What has been presented so far establishes beyond doubt that it is possible to disclose a classical spaceframe structure for the double stringed elementary particle spectroscopy, in which the nucleon holds pivotal position, the mesons are the pylon sections and the leptons their beam and suspension elements. The warp, the string, the knots, the pattern; all comply, but how to weave the tapestry: the Atom that is ten thousand times larger? It must be by filling it by the same stuff because spacefilling goes with the provision of three dimensions alone where the self-referential filament is the sole thread (or string) available and allowed. This holds also when there are more dimensions because our distinct Universe still consists only of itself and then also consummates itself and as the three spatial dimensions in it are linearly independent the situation prevails in their subgroup. Since the spaceframe grid is infinitesimal at the elementary particle threshold, there are no loopholes, and the expansion must go on by it, so the atom can only be a periodical enlargement of its arrangement in order to accommodate in the global coherence. 


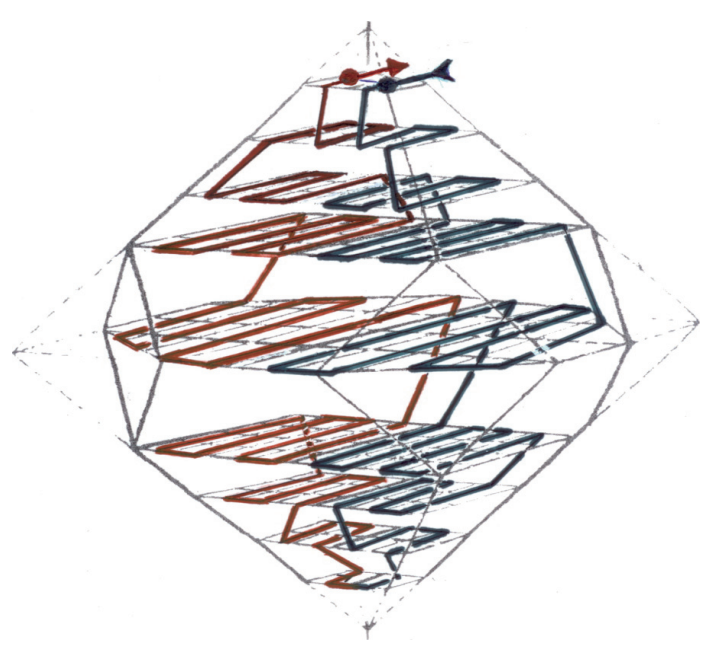

Fig. 24. One possible, net Fermion continuous sequence of singlet rosettes in Hydrogen electron module connecting with likewise Fermion proton root vector at the origin forming the net Boson Hydrogen atom. The module tiers correspond to the Bohr orbital shell and hold same numbers of rosettes as the electrons there; also in the $\mathrm{P}$ to $\mathrm{S}$ levels where higher amounts have not been seen in reality.

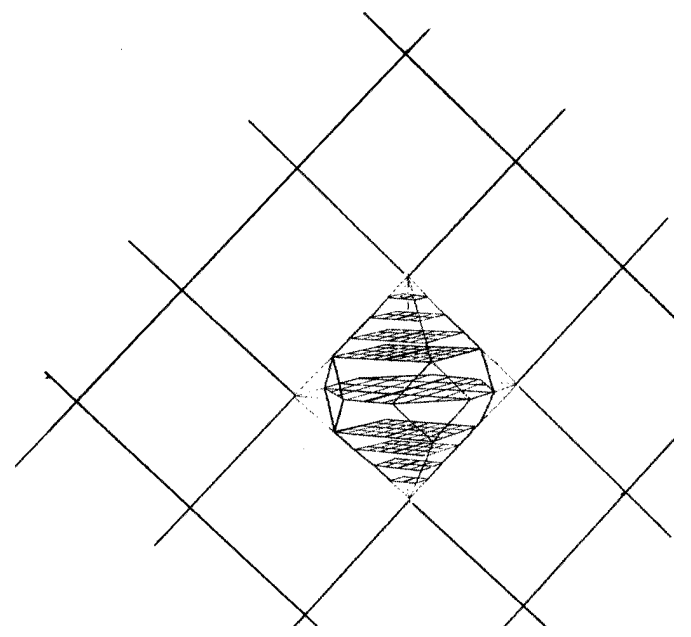

Fig. 25. The electron module is surrounded by other modules in the second-generation global lattice, and thus doubly bound to its segmental shape.

With the leptons all elementary particles are reproduced by an instantaneous principal phase transition where the electron cloud in one variety pursued here comes out as a spacefilling mesh segment of defined, second-order regular solid form. This truncated octahedron module can be seen as a diagonal cube possible to tessellate into different shapes which, in turn, may self-template into cyclically larger portions of same or modified form to go on filling space in a three-dimensional Tetris way, and, at any such stage, to combine with each other in various full-packing conformations (Fig. 25).

Figure 26 shows that the diagonal arrangement allows accommodation of separately counter-spinning modules with corresponding implications for the formation of larger regions in a cyclical mushrooming way.

There is nothing different from the orbital model in that regard, under one crucial provision: that the continuous transition lattice can also be continuously delineated. Figure 24 verified that this is indeed the case under a Fermion half-spin rotation around the forward diagonal axis bringing the end of the line one charged root vector step and one or two (or at lowest quantum, Bose-Einstein Condensate state, zero) neutral space axis steps from the origin which, not taking part in the electron formation, appears as the reciprocal pivot, each point of which is 1852 times longer lasting than the electron with proportionately higher inertia and consequential mass number: $1852 \times 0.514=938.27 \mathrm{MeV}$. The advantage is that the distribution solid can be used as structural bricks, and this double cast of the electrons as "wave functions or transition matrix elements" is in line with recent Hydrogen ground state research [35] and the instant material "modular building block" [36] nature of the electron is pending in modern nanotechnology, molecular biology etc. However other configurations are also possible, but the main idea is to show that it is possible to find a continuously space-filling three-dimensional modular electron plot.

Figure 27(a) illustrates the complex of one electron module linking with the proton in an upper Cartesian segment and so matching the Hydrogen atom. At this stage the deep relation to Santilli's revolutionary hadronic mechanics $[37,38]$ and its reduction of matter to, and from, the proton and electron 


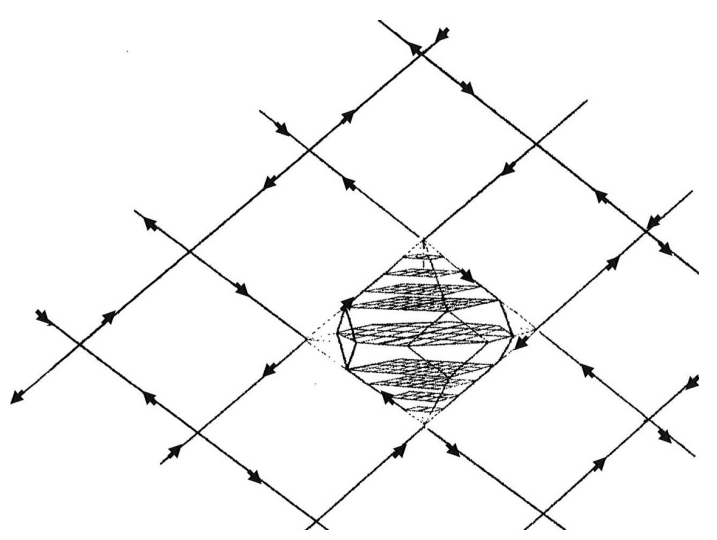

Fig. 26. The electron module can be accommodated with other modules and also with continuous expansions of itself.

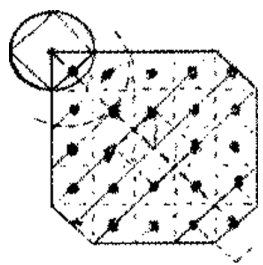

(a)

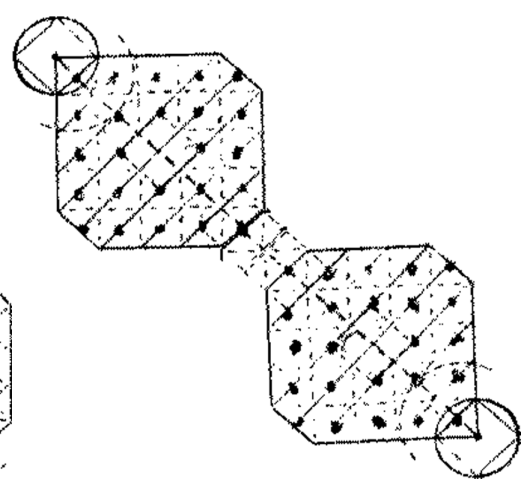

(b)
Fig. 27. a) Horizontal plane projection of single extranucleon module with open end and so realizing $\mathrm{H}$. b) When two $\mathrm{H}$ ions are linked end-to-end (or side) the $\mathrm{H}_{2}$ molecule is formed.

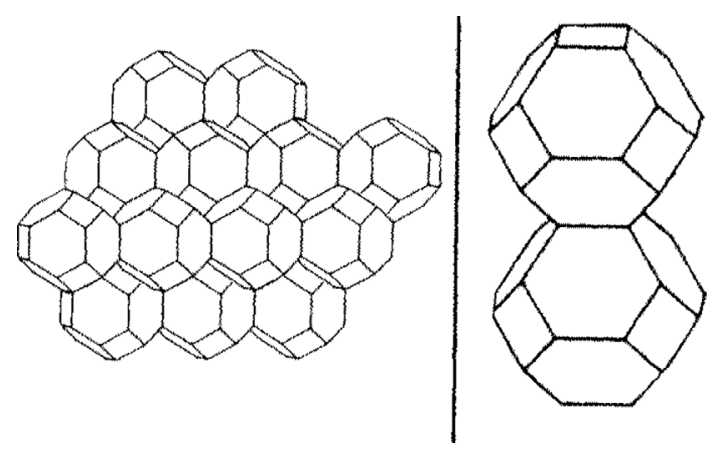

Fig. 28. Honeycombs of truncated octahedrons and of the Helium atom.
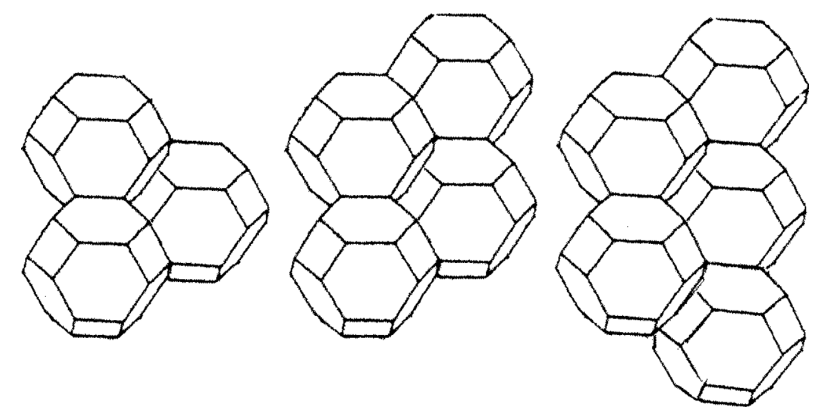

Fig. 29. The Lithium (one Module in L sheet), Beryllium (2 L modules) and Boron (3 modules) Electron Honeycombs.

becomes apparent, because the complex persists in the successive levels of penetration, from the union in the nucleon domain via the muon trajectory and decay channels over its surface to the continuously spacefilling module expansions outside, whose first cycle is depicted in the figure.

Figure 27(b) shows that the opposite end of the proton-electron complex is free to bind with another open-ended ion, here a second $\mathrm{H}$ into the $\mathrm{H}_{2}$ molecule. It is a variety of "nested polyhedra... which can in turn be put together in spatial arrangements", e.g. "helicoidal progression" [39]; in the present case creating the Bohr orbit signature of the singlet nodes in the forward plane. And when instead under strong pressure two Hydrogen ions will fuse so that one is pushed a step upwards, still rooting with the upper proton pole in the nucleon and the other with the under and thereby also the in-between neutrons' space axis points are involved, a two-module truncated octahedron honeycomb is generated (Fig. 28), closing the ground $(\mathrm{K})$ sheet of lattice intersections and therefore very stable so as to faithfully realize the Helium atom.

In that way the singlet sites can be dragged in under an expanding central boundary as nucleon centres of consecutively larger honeycombs, which thereby are templated in steps and constellations of the periodical system and onwards to further self-similar space-filling, for instance, of crystalline lattices, deposits, rocks, planets etc. Exemplifying the mechanism only in the first three atoms from the next (L) sheet, the Lithium honeycomb is variably triangular with one free end for molecular coupling whereas 


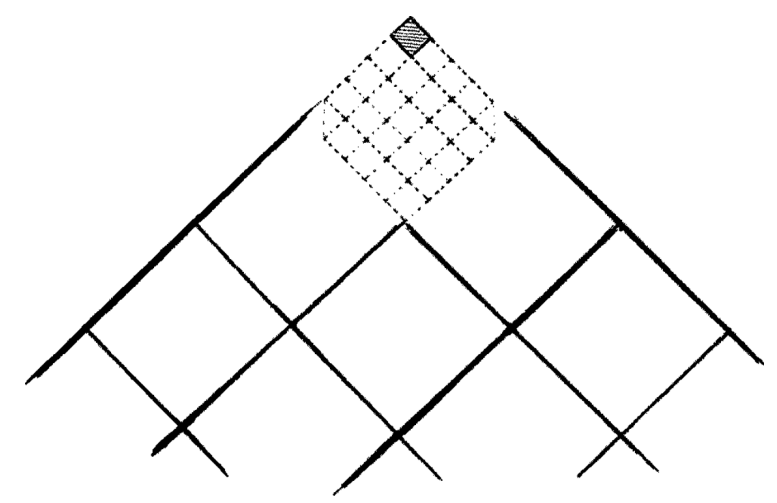

Fig. 30. Schematic equatorial plane projection of first three self-similar cycles of electron module in the Hydrogen atom as well as of the vertically doubled (Fig. 26) Helium atom.

the square or rhombic Beryllium can combine with two atoms/ions/complexes and Boron with three (Fig. 29).

Not illustrated, Carbon can permutatively couple/chain with four including itself, whereas Nitrogen holds five of the L positions and so has three to offer; Oxygen then two, and Fluorine very strongly one; and when the $\mathrm{L}$ shell is filled a new saturated and hence stable atom, Neon, is established.

And so it continues and the correspondences are so extensive, and also non-trivial, that there can be little doubt that it is along these principles that matter will be ultimately reconstructed in forthcoming nanotechnology. For instance, when one considers the situation meeting individual Hydrogen ion electron modules in the interior of large stars, it is easy to imagine how the enormous forces there squeeze out their coils to axial or crisscross meandering cords of the nucleon core clustered shell-wise parallel [40] or twisted around each other so that when the physical conditions get less intense they cycle by cycle regain their electron mantle, now caught in the atomic bouquet corresponding to the number of modules wrung together. Exactly where an observation instrument head-on intercepts such a cross-section is a matter of quantum indeterminacy and probability statistics. And so far the reproductions here only comprise the first extranucleon level, or quantum state of the elements. This is the situation prevailing in the Bose-Einstein condensation but according to the aforesaid also at the other extreme of the temperature scale [41] where the Hydrogen ion modules enter into the fusion cascade.

Therefore, it is group-theoretically relevant and interesting that as an inverse at the other, zero Kelvin end of the temperature range, the charged root vector suit runs back into empty space, that is, into the rectilinear lattice moiety by the same route it appeared. This can only be by a honeycomb singlet, which can come so close whereas larger constellations are too distanced. But also in the singlets there is a difference, first exhibited by the Fermion Hydrogen ion which comes back $1 / 2$ step along the projection planes away from the origin so that retrograde access to the Euclidean space is blocked as it were, whereas the larger Boson Helium will come at +1 and so hitting the entrance; which Hydrogen can do by pairing [41]. Similarly, the Fermion Lithium settles at $1^{1 / 2}+1^{1 / 2}=3$, while the Boson Beryllium arrives at 2 at once... and so on in the same pattern as observed in experiments throughout the periodic system. [Ib.] Apart from supporting the faithfulness and pertinence of the regular solid scheme it also illustrates that perfect precision, which after all characterises clarified physical reality [20,42], can only run through all magnitudes of this if generated already at the outset by the not only perfect but categorical precision of an absolutely unique and universal quantum phase transition; the only that exists per se by itself and thus guaranteeing its perpetuation. 
The Bose-Einstein condensate stage is also interesting because of its intermediary state at the entry of the channel between interior and exterior so that the experimental observations are paralleled and even aided by the hadronic mechanical and present faithful structural charts of the route of partial reversibility at the threshold. When proceeding from the singlet honeycombs to the atoms there is a cyclical expansion of the respective basic motif which can be described as a stacking of exponentially larger boxes in a Tetris-like manner, each generation templated by the previous along adapted route that worked in the preceding ones so that the single electron geodesics remains unbroken. The number of generations is then, as cause and effect, temperature-dependent. From just one at both the hot and cold extremes, it increases towards the logarithmic mean which for many reasons would be around where water flows and life is formed, and where, as also in other quantum levels, the equal Avogadro pressure of equally many atoms (in gaseous state) reflects the proportional number of root vector steps in their completion. Figure 30 shows the principle in a horizontal plane projection towards three cycles in the Hydrogen atom stacking, and it is seen that it does not take many cycles before the cross-section is zoomed up ten-thousand-fold as is also the ratio between the actual elementary particles and atoms.

Interestingly, then, the atom region is outlined by the Electron cloud which is spacefilling and in that sense complying with the quantum mechanical fact that there is a probability of finding the single electron anywhere in the cloud, and that it is the instrumentation interaction that determines the recorded features of it; notably its size. As commonly regarded it is "a pointlike particle-that is, a particle with no measurable dimension... However, a rather compelling case can be made for an opposing viewpoint: namely, that the Electron is in fact a large particle which contains an embedded point-like charge" [43]. This complies well with the findings here that at the bottomline stage all elementary particles are of the same size order since spanned by the same digital bit. Its length, as stated in hadronic mechanics, too [38], is that of the Proton radius $(\approx 0.8768 \mathrm{fm})$.

Here, the transition matrix of the Electron cloud has been approximated with the truncated octahedron, but other continuous polygonal realizations are possible, too, and perhaps more likely to more clearly account for the nucleon. That also the large-scale arrangement of matter in the flat Universe follows a polyhedral plan is supported even on the astronomic level [44,45], and seems to consist of the same mix of octahedrons and tetrahedrons that appear already on the infinitesimal plane in the present scheme (Figs $1-3,8,14,15,23,24)$.

Single as well as fused in honeycomb and molecular aggregates, the modules heap up the joint structural architecture as veritable Lego pieces, patching together already at the infinitesimal level every three-dimensional real shape from their consecutive own and composed combinations. This does not mean that they are some static wire bundles, but the second-generation, $2^{3 \times 3}$ periodical partition of the continuous space-filling charged root vector lattice into the first self-similar extra-nuclear segment of the global transition matrix. Its outline may be distended in, for instance, accelerations, but then the surrounding modules, whether occupied or empty at the moment, will, too, and the apportioned volume share remains preserved. One possible sequential ordering of the electron singlet subunits (Fig. 24) runs through the (here) upper Cartesian segment from its origin and returns in the one below, and so gradually shifts the proton one unit step down and changes the module progression to the opposite direction so as to describe a virtual cross-section rotation with Bohr orbital signature. And when in larger atoms their respective nuclear hub extends over a larger domain of singlets, which in turn magnify their (sometimes isotopically varying) constellation to the honeycomb they co-ordinate, the interstitial charged and neutral root vector content in them will manifest as the corresponding atomic number of protons and neutrons.

For instance, since the electron geodesic is wrapped throughout the entire atom it matches the "quantum superposition... qualitative picture of all possible electron paths conspiring together" [46] with 
correspondingly low probability of hitting it in a particular infinitesimal interaction cone. And the propagation of the atoms themselves when they occupy their consecutively inflated domains would be determined by their template form so that highly symmetrical shapes, like the noble gases, would proceed in one-dimensional curves and accordingly be gaseous while sharply bent honeycomb modules, like Lithium, regardless of its low weight would go into dense, net two- or three-dimensional convolutions so as to be solid (until heated/excited so that it starts to boil into orbit). And since the offset 'caps' that the honeycombs' collective truncation leaves at the top contain the abandoned central isospin vectors there will be a reciprocal nucleus, always with as many charged, proton roots as the atomic number, while the Neutrons can be more numerous reflecting the lateral displacements possible under acceleration [47], e.g. in ${ }^{11} \mathrm{Li}$.

\section{Discussion}

Peter Rowlands has pointed out that since the algebraic solutions in his Nilpotent Universal Computer Rewrite System (NUCRS) as well as the present morphological results "are based on the same theory (QCD), then we should expect a correspondence" [10]. There is a double endorsement then: being strongly endorseded by this leading theory, the findings offer equally strong support back to it by endowing it with a direct real structure capable to build both itself and its act and "stage itself... on the smallest length and time scales... (where)... just as matter... space consists of tiny individual bits" [2] which are genuinely irreducible since they are the simplest of elements that there can be; infinitesimal straight line slices, stepping immediately out into a naturally digital existence from the total antithesis of this, namely, complete oblivion; The absolute eigenvectors both ontologically and substantially of anything-at-all versus its categorical contrast of nothing-whatsoever. Because nothing-whatsoever is not a void, is not an empty space because space is extension, while nothing-whatsoever is absence and absence also of extension and so shrinks away to a deep down impossible contradiction in terms against which presence, that there is something, stands forth as an equally categorical and bare option whose limitlessness goes in the other direction as infinite extension with the endless straight line as instant physical embodiment-and by that effective immanence of something the nothingness alternative is disproved in the passing.

When it comes to the actual construction work, the raw sketches here need a lot of further development. Nonetheless it can be discerned that they point to a general procedure from which the full inorganic realm and its likewise regularly polygonal macroscopic minerals and crystals can be reproduced with all the attributes of the periodic system. It has been a very condensed survey, focussed on the reproducible descriptive results and it stops at the atom stage, but can be extended over molecules and larger compounds to the astronomical scale $[44,45]$. Like many other current models, it is a lattice system, however, almost embarrassingly simple in comparison, perhaps too simple to be true. Yet, it has been said that there is a crisis in today's physics [48], so that the merit lies in the concrete, up-to-date nanotechnology outcome as well as in the eminent 'back to the future' legacy: such as the eightfold way, the Lie groups and algebras, the NUCRS, the Lie-admissible hadronic mechanics, the Diophantine equations and, first and not least, the ancient regular solids, of which in the inorganic field here dealt with only the static cube of the geocentrically inferred space matrix and the tetrahedron and the octahedron of the dynamic brew of fire and air [20,21], respectively, are employed. They are formed by successive straight line steps from the infinitesimal stage; rendering them genuine solids, and also implying that at the physical ground nothing else exists or even needs to exist-implying, finally, that universe is self-similarly filled entirely by its own being. 
It is argued, that the straight line is a direct structure and digital bit, but also the irreducible vector element of pure existence, of anything at all. It is also as the binary impulses and synapse branching of the neuronal system the element and operator of intelligence, logic and language as shown by Johansen in his ground-breaking Differential Epistemology [49]. A related, more anthropic argument for straight is that we and our perceptions are directly parts of and resonating with reality all from the quantum level. In other words, we should pay much attention to testimonies like the following (cited from Tate Modern): "Piet Mondrian (1872-1944) believed that all complex forms could be reduced to a "plurality of straight lines in rectangular opposition'... his paintings... also represent a physiological reality about the brain... the cells of the visual brain are responsive to straight lines of specific orientation and the field of view to which they respond is rectangular in shape".

However, the strongest argument is the reproducible outcome. It is obtained by genuine first principles and in many instances comprises a first itself. And the results are what counts and persists; Some day, some model will prevail, and the simpler and more akin to the world at large the better and more plausible and workable. At the elementary particle/atomic stage the direct structural embodiments here cover the inorganic realm with unprecedented resolution and completeness, including-wherever properly looked for-entanglement as well due to the overall coherence of the literally bottom-line lattice, whose systematic linkage of a ground irreducible linear bit is seen to be capable of self-aggregating the exhaustive type-set for printing out, as it were, the entire primer of real matter. But the second edition, the textbook of organic Nature, requires a larger alphabet built upon and from molecular combinations of this atomic substrate in a predictable evolutionary permutation way so as to preserve nanotechnological precision and intertwining organisation in order to assemble and accommodate, for instance, "Salmonella's needle complex" in subnanometer sharpness [50] and the fragile "beauty in complexity" of intricate "molecular motors" [51]. It is fascinating to consider that in their multiplied scale such protein components still recruit and utilise the regular solids, now also including the making and makings of pentagonal and mixed symmetry dodecahedrons and icosahedrons, to grandiose constructions of minute detail, doubtlessly enabling in the not-too-distant future total charts of equal extensiveness and exactitude as a racing car's explosion diagram. Fundamental morphological work in this direction employing concrete structural modelling is under way above all by Hill and Rowlands [10,52].

In conclusion, the present paper is but a brief summary in need of further clarification. Especially, this applies to the question whether the truncated octahedron and the honeycombs it can put together really are the building elements of the continuous atom delineation. The electron singlet rosette (Figs 2,14,15) is an octahedron/tetrahedron complex and it seems more likely that if it isomorphically templates the ensuing period of its form it would be by iterating this composition in the half-turn twist because "if this is done in the same way as the faces... a second generation of polyhedra is formed, which takes the place of the polygonal faces of the first. This can be done repeatedly" [39] with multiplicative enlargement of the motif, that can be carried forth in a helical propagation (Ib.), conveying the Nucleon hub along its eccentrically dilating spiral whereas the honeycomb stacking doesn't have a centre of revolution. A suitable floral prototype which will be focussed on in the forthcoming, stage of the pursuit of the (crosssection) of the atom world-line is then posed by the sunflower. Like the double spiral iteration over its bed of identical individual flowerets in a Fibonacci series arrangement, the matching atomic distribution would be grainy, too. For every increased atomic number another single proton-neutron nucleus adds to the chain and a new electron module of automatically the same period as in the lower states adjoins. In regard of the synthesis and "packing of nucleons", this astonishingly simple arithmetical configuration is akin to the "understanding of atomic matter through number theory" disclosed by Boeyens and Levendis [53], and in principle compatible as well with the discrete quantum organization of the Quark model. But it differs by the direct structural rendering. 
This reaches also into the nucleon as the residue of the electron phase transition outside it, which in every 'rosette' (Figs 14 and 15) consummates the 12 unit sides of the cube it replaces, either in a static closed loop (Fig. 14) or a dynamic open loop by translation of the last step (Fig. 15). Whereas the former variety stalls the whole process, the latter drives the continuation into the periodical expansion of the motif as suggested in Figs 24 and 30, with reciprocal consequences for the root vectors remaining inside. For each electron module mushrooming from the surface of the nucleon there is one unit vector of opposite charge left in its interior from the centre (Figs 2 and 3), i.e. the proton, and when the module goes into its next 1852-step revolution this digital unit is moving one step along its charge axis (Fig. 24). Likewise, there is a movement over the unit side vectors of the Euclidean space encasement, i.e. the neutrons, which depending upon the winding course of the convoying hub procession tend to outnumber the protons, in higher mass number nuclide bouquets closing in upon the golden ratio [53]. However, all the way it remains a strictly digital system replicating the likely sequence of events in the Cosmogenesis: first there is the instant isotropic inflation of the hydrogen gas simply by is taking place by the dynamic phase transition (Fig. 15), then occasional internal encounters in the gas with increasing pressure and temperature squeezes more and more of the ions together, so that ultimately the nucleogenesis era is ignited, when layer after layer of the electron modules in increasingly compressed ions are peeled off by their sequences unwound under ejection of photon and neutrino strings until their core is reached and they can, templating their own Bohr layout, gather and align in that pattern like the axons of a nerve fiber or strands of an electric cable. . . and when cooling off accumulate electronically again to composite atoms packed in such distributed manner till at zero K they might be singly or pair-wise squeezed back as Bose-Einstein condensates to interaction-less neutral stand-still. . . as in reality.

\section{References}

[1] A.H. Guth and D.I. Kaiser, Inflatory cosmology: Exploring the universe from the smallest to the largest scales, Science 307 (2005), 884-890.

[2] A. Cho, Constructing spacetime-no strings attached, Science 298 (2002), 1166-1167.

[3] E. Trell, A calculation of the electron circular orbital radius, Speculation in Science and Technology 5 (1982), 533-535.

[4] E. Trell, Representation of particle masses in hadronic SU(3) diagram, Acta Physica Austriaca 55 (1983), 97-110.

[5] E. Trell, On rotational symmetry and real geometrical representations of the elementary particles with special reference to the N and $\Delta$ Series, Physics Essays 4 (1991), 272-283.

[6] M.S. Lie, Over en classe geometriske transformationer, Ph D Thesis, Kristiania (now Oslo) University, 1871.

[7] E. Trell and R.M. Santilli, Marius Sophus Lie's doctoral thesis Over en classe geometriske transformationer, Algebras Groups and Geometries 15 (1998), 395-445.

[8] E. Trell, The eightfold eightfold way: Application of Lie's true geometriske transformationer to elementary particles, Algebras Groups and Geometries 15 (1998), 447-471.

[9] E. Trell, The eightfold eightfold way: A lateral view on the standard model, Physical Interpretations of Relativity Theory (11-14 September 1998), Late Papers, London: British Society for the Philosophy of Science, (2000), 263-284.

[10] P. Rowlands, Zero to infinity: The foundation of physics, World Scientific (2008).

[11] R.L. Jaffe, Quark confinement, Nature 268 (1997), 201-208.

[12] E. Trell, Geometrical reproduction of (u,d,s) baryon, meson, and lepton transformation symmetries, masses, and channels, Hadronic Journal 13 (1990), 277-297.

[13] E. Trell, Real forms of the elementary particles with a report of the $\Sigma$ resonances, Physics Essays 5 (1992), $362-373$.

[14] E. Trell, Real charm of form-real form of charm. Duality in transition. in: Fundamental Open Problems in Science at the End of the Millenium, T. Gill, K. Liu and E. Trell, eds, Palm Springs: Hadronic Press, 1999, pp. 1-29.

[15] E. Trell, String and loop quantum gravity theories unified in platonic ether. With proof of Fermat's last theorem and Beal's conjecture, in: Proceedings of International Scientific Meeting PIRT - 2003, M.C. Duffy, V.O. Gladyshev and A.N. Morozov, Moscow 30 June-03 July, 2003, Bauman State University, Moscow, Liverpool, Sunderland, 2003, pp. 134-149.

[16] E. Trell, Classical 3-d. geometrical 'vortex sponge' world-ether provides natural quantum cavity elementary particle standing wave incubation and original diophantine equation encapsulation, Physical Interpretations of Relativity Theory IX (3-6/9 2004), Proceedings, London: British Society for the Philosophy of Science, (2004), 503-30. 
[17] E. Trell, Invariant aristotelian cosmology: Binary phase transition of the universe from the smallest to the largest scales, Hadronic Journal 28 (2005), 1-42.

[18] E. Trell, An excursion in and betweem curvature II. From classical lie algebra neighbourhood to QED and QCD of real elementary particles, Bull Cal Math Soc 97 (2005), 573-588.

[19] E. Trell, Filling a gap in nilpotent vacuum: How close are we to a fundamental reality?, Physical Interpretations of Relativity Theory X (8-11 September 2006), Proceedings, London: British Society for the Philosophy of Science, 50330.

[20] E. Trell, Back to the ether, in: Ether Spacetime \& Cosmology, (Vol. 3), M.C. Duffy and J. Levy, eds, Montreal: Apeiron, 2009, pp. 339-387.

[21] E. Trell, Elementary particle spectroscopy in regular solid rewrite, American Institute of Physics Conference Proceedings 1051 (2008), 127-41.

[22] L. Hogben, London: Georg Allen \& Unwin, Mathematics for the Million (1937).

[23] E. Trell, Classical information matters - and applies, Applied Mathematics \& Information Sciences (2011).

[24] M. Kamionkowski, A Hawking-eye view of the universe, Science 296 (2002), 267.

[25] H. Kleinert, Gravity as a theory of defects in a crystal with only second gradient elasticity, Annalen der Physik 44 (1987), $117-119$.

[26] E. Trell, An alternative solution to fermat's last theorem: Infinite ascent in isotopic geometry, Hadronic Journal Supplement 12 (1997), 217-240.

[27] E. Trell, Isotopic proof and reproof of Fermat's last theorem verifying Beal's conjecture, Algebras Groups and Geometries 15 (1998), 299-318.

[28] E. Trell, Original diophantine equations lodge BC without ABC, International Symposium on Recent Advances in Mathematics and its Applications, Proceedings, Calcutta: Calcutta Mathematical Society, 2003.

[29] E. Trell, Original diophantine equations lodge BC without ABC, Rev Bull Cal Math Soc 12 (2004), 29-54.

[30] E. Trell, Cubit isounits 'tread a daunting path to reality' while proving Fermat's last theorem and Beal's conjecture, Hadronic Journal 26 (2004), 237-271.

[31] E. Trell, Tessellation of diophantine equation block universe, Physical Interpretations of Relativity Theory VIII, Proceedings, London: British Society for the Philosophy of Science, (2002), 585-601.

[32] E. Trell, An excursion in curvature I. Diophantine equations get real again in re-established flat euclidean space, Bull Cal Math Soc 97 (2005), 551-572.

[33] E. Trell, Scheme for a time antenna in three-dimensional hausdorff space, Speculations in Science and Technology 7 (1984), 269-277.

[34] E. Trell, Temporospatial transition - back to go, Physical Interpretations of Relativity Theory (15-18 September 2000), Late Papers, London: British Society for the Philosophy of Science, (2004), 305-311.

[35] F. Martin, J. Fernández, T. Havermeier, L. Foucar et al., Single photon-induced symmetry breaking of $\mathrm{H}_{2}$ dissociation, Science 315 (2007), 629-633.

[36] O.L. De Weck, W.D. Nadir, J.G. Wong, G. Bounova and T.M. Coffee, Modular structures for manned space exploration: The truncated octahedron as a building block, AIAA 2005-2764 (2005), 1-26.

[37] E. Trell, Book review: Foundations of hadronic chemistry with applications to new clean energies and fuels, International Journal of Hydrogen Energy 28 (2003), 251-253.

[38] I. Gandzha and J. Kadisvily, New sciences for a new era, Mathematical Physical and Chemical Discoveries of Ruggero Maria Santilli, Kathmandu: Sankata Printing Press, 2011.

[39] P. Huybers, Nested polyhedra, IASS Newsletter 14 (2007), 31-40.

[40] J.C.A. Boeyens and D.C. Levendic, Number theory and the periodicity of matter, New York: Springer, 2007.

[41] A. Cho, Insights flow from ultracold atoms that mimic superconductors, Science 319 (2008), 1180-1181.

[42] M. Schmidt and H. Lipson, Distilling free-form natural laws from experimental data, Science 324 (2009), 81-85.

[43] M.H. Mac Gregor, The Enigmantic Electron, Dordrecht: Kluwer, 1982.

[44] C. Seife, Polyhedral model gives the universe an unexpected twist, Science 302 (2003), 209.

[45] E. Battener and E. Florido, The egg-carton universe, arXiv:astro-ph/9802009v1, (1998), 1-7.

[46] J. Ambjørn, J. Jurkiewics and R. Loll, The self-organizing quantum universe, Scientific American 6 (2008), 42-49.

[47] B.M. Sherrill, Designer atomic nuclei, Science 320 (2008), 751-752.

[48] A. Cho, Does Fermilab have a future? Science 320 (2008), 1148-1151.

[49] S. Johansen, Grunnriss av en differensiell epistemologi, Bergen: Ariadne, 2009.

[50] O. Schreidt and Th.C. Marlovits, Three-dimensional model of salmonella's needle complex at subnanometer resolution, Science 331 (2011), 1192-1195.

[51] J.A. Spudich, Molecular motor: Beauty in complexity, Science 331 (2011), 1143-1144.

[52] V.J. Hill and P. Rowlands, Nature's code, American Institute of Physics Conference Proceedings 1051 (2008), 127-126.

[53] J.C.A. Boeyens and D.C. Levendis, Number theory and the periodicity of matter, New York: Springer, 2007. 